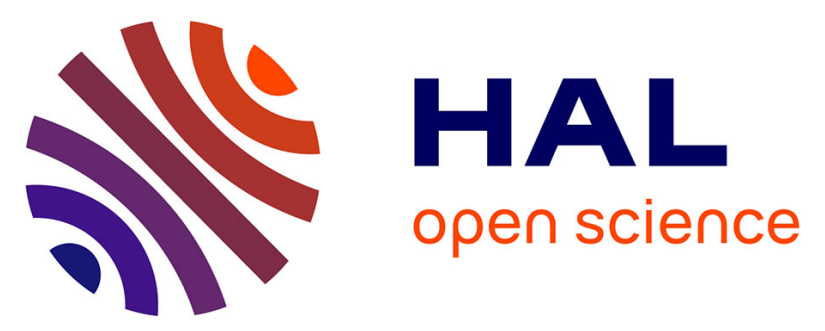

\title{
Cortical surface mapping using topology correction, partial flattening and 3D shape context-based non-rigid registration for use in quantifying atrophy in Alzheimer's disease.
}

Oscar Acosta, Jurgen Fripp, Vincent Doré, Pierrick Bourgeat, Jean-Marie Favreau, Gaël Chételat, Andrea Rueda, Victor L. Villemagne, Cassandra Szoeke, David Ames, et al.

\section{- To cite this version:}

Oscar Acosta, Jurgen Fripp, Vincent Doré, Pierrick Bourgeat, Jean-Marie Favreau, et al.. Cortical surface mapping using topology correction, partial flattening and 3D shape context-based non-rigid registration for use in quantifying atrophy in Alzheimer's disease.. Journal of Neuroscience Methods, 2011, 205 (1), pp.96-109. 10.1016/j.jneumeth.2011.12.011 . inserm-00664033

\section{HAL Id: inserm-00664033 https://www.hal.inserm.fr/inserm-00664033}

Submitted on 1 Feb 2012

HAL is a multi-disciplinary open access archive for the deposit and dissemination of scientific research documents, whether they are published or not. The documents may come from teaching and research institutions in France or abroad, or from public or private research centers.
L'archive ouverte pluridisciplinaire HAL, est destinée au dépôt et à la diffusion de documents scientifiques de niveau recherche, publiés ou non, émanant des établissements d'enseignement et de recherche français ou étrangers, des laboratoires publics ou privés. 


\title{
Cortical surface mapping using topology correction, partial flattening and $3 \mathrm{D}$ shape context-based non-rigid registration for use in quantifying atrophy in Alzheimer's disease
}

\author{
Oscar Acosta ${ }^{\mathrm{a}, \mathrm{m}}$, Jurgen Frippa, Vincent Dorea, Pierrick Bourgeata, Jean-Marie Favreau ${ }^{\mathrm{b}}$, Gaël \\ Chételatc,d, Andrea Ruedaa,e, Victor L. Villemagne ${ }^{d}$, Cassandra Szoekea, David Amesi, Kathryn A. \\ Ellis, Ralph N. Martinsk, Colin L. Masters ${ }^{\mathrm{f}, \mathrm{g}}$, Christopher C. Rowe ${ }^{\mathrm{d}, \mathrm{h}}$, Erik Bonnera, Florence Gris ${ }^{\mathrm{a}}$, Di \\ Xiao $^{a}$, Parnesh Ranigaa, Vincent Barrab, Olivier Salvadoa \\ aCSIRO Preventative Health National Research Flagship, ICTC, \\ The Australian e-Health Research Centre - BioMedIA, Herston, Australia. \\ bLIMOS, UMR 6158 CNRS, Université Blaise Pascal, Aubière, France \\ cINSERM-EPHE-Université de Caen/Basse-Normandie, Unité U923, GIP Cyceron, CHU Côte de Nacre, Caen, France \\ dDept of Nuclear Medicine and Centre for PET, Austin Health, VIC, Australia \\ eBiolngenium Research Group, Universidad Nacional de Colombia, Bogotá, Colombia, \\ The Mental Health Research Institute, The University of Melbourne, VIC, Australia \\ gCentre for Neuroscience, The University of Melbourne, VIC, Australia \\ hDept of Medicine, Austin Health, University of Melbourne, VIC, Australia \\ 'National Ageing Research Institute, Melbourne, VIC, Australia \\ jAcademic Unit for Psychiatry of Old Age, Department of Psychiatry, The University of Melbourne, St. Vincents Aged \\ Psychiatry Service, St Georges Hospital, VIC, Australia \\ ${ }^{k}$ Centre of Excellence for Alzheimers Disease Research Care, School of Exercise Biomedical and Health Sciences, Edith \\ Cowan University, Joondalup, WA, Australia \\ IUniversité de Rennes 1, LTSI, Rennes, F-35000, France \\ mINSERM, U642, Rennes, F-35000, France
}

\begin{abstract}
Magnetic resonance (MR) provides a non-invasive way to investigate changes in the brain resulting from aging or neurodegenerative disorders such as Alzheimer's disease (AD). Performing accurate analysis for population studies is challenging because of the interindividual anatomical variability. A large set of tools are found to perform studies of brain anatomy and population analysis (FreeSurfer, SPM, FSL). In this paper we present a newly developed surfacebased processing pipeline (milxCTE) that allows accurate vertex-wise statistical comparisons of brain modifications, such as cortical thickness (CTE). The brain is first segmented into the three main tissues: white matter, gray matter and cerebrospinal fluid, after CTE is computed, a topology corrected mesh is generated. Partial inflation and non-rigid registration of cortical surfaces to a common space using shape context are then performed. Each of the steps was firstly validated using MR images from the OASIS database. We then applied the pipeline to a sample of individuals randomly selected from the AIBL study on AD and compared with FreeSurfer. For a population of 50 individuals we found correlation of cortical thickness in all the regions of the brain (average $r=0.62$ left and $r=0.64$ right hemispheres). We finally computed changes in atrophy in $32 \mathrm{AD}$ patients and 81 healthy elderly individuals. Significant
\end{abstract}


differences were found in regions known to be affected in AD. We demonstrated the validity of the method for use in clinical studies which provides an alternative to well established techniques to compare different imaging biomarkers for the study of neurodegenerative diseases.

Keywords: Cortical mapping; Surface registration; Partially inflated surfaces; Cortical thickness; Brain Atrophy; Shape context; Statistical analysis of populations ; Alzheimer's disease; Freesurfer 


\section{Introduction}

Non-invasive imaging techniques such as magnetic resonance imaging (MRI) allow the investigation of many of the functional and morphological changes in the brain occurring with age or during pathological processes. However, reliable statistical analysis of available data over a large number of individuals are required before detecting subtle differences over time or between individuals and groups. The distribution differences of specific tissues such as white matter (WM), gray matter (GM) or cerebrospinal fluid (CSF), volume changes, or any other signal coming from imaging devices or intermediate pre-processing stages have been used in the past, unravelling some of the complex pathological changes. Statistical analysis per region (Lerch et al. 2005), voxels (Ashburner and Friston 2000), (Ziolko and textitet al 2006) or vertices in a surface representation of the brain (A.M. Dale, B. Fischl, and M.I. Sereno 1999), (B. Fischl, M. I Sereno, and A. M Dale 1999) can be used to assess the differences between groups using, for instance, a general linear model (GLM) to the available data (intensity distribution, deformation, etc.) from all subjects at each region (voxel or vertex) with different covariates such as age, years of education, gender, diagnosis, cognitive scores, etc. Some of these techniques have quantitatively demonstrated different biomarkers or predictors for Alzheimer's Disease (AD), showing a specific pattern of atrophy (Hua et al. 2008), (Querbes et al. 2009), hypometabolism (Chételat et al. 2007), $\beta$-amyloid load (Mikhno et al. 2008) or combined hypometabolism and atrophy (Kawachi et al. 2006), (Villain et al. 2008) and sulcal modifications (Zhan et al. 2009) using MRI or Positron Emission Tomography (PET).

Different software tools, grouping a wide range of methods to perform studies of cortical and subcortical anatomy are nowadays available. Most of them are also able to carry out further population analysis. Statistical Parametric Mapping (SPM) ${ }^{1}$ or FreeSurfer (FS) ${ }^{2}$ represent two of those automated tools largely referenced in the literature. Although they

\footnotetext{
${ }^{1}$ http://www.fil.ion.ucl.ac.uk/spm/

${ }^{2}$ http://surfer.nmr.mgh.harvard.edu/
} 
share the same goals, the comparisons are performed in different ways. SPM is a voxel-based approach in which the images are realigned, spatially normalised into a standard space, and smoothed before performing statistical analysis at a voxel level (Ashburner and Friston 2000). In contrast, FreeSurfer is a surface-based approach performing first the reconstruction of the brain cortical surface from structural MRI data (B. Fischl, M. I Sereno, and A. M Dale 1999). FreeSurfer provides many anatomical analysis tools, including the representation of the cortical surface, segmentation of the brain, skull stripping, bias field correction, nonlinear registration of the cortical surfaces into a standard space, labelling of regions and statistical analysis of populations. Comparison of those different tools has been performed in the past for specific tasks such as segmentation (Klauschen et al, 2009). Overall, they face similar methodological challenges for interindividual comparisons.

The primary issue for interindividual comparison is the mapping or registration of a given population to a common space. This is a particularly challenging task because of the brain convoluted geometry and the high interindividual variability (J.-F. Mangin et al. 2004). Various registration approaches have been proposed, including intensity-based in the voxel space (Rueckert et al. 1999), (T. Vercauteren et al. 2007) or shape-based in a surface representation of the brain (Yeo et al. 2008, 2010), (Eckstein et al. 2007). Although intensity-based non-rigid registration methods work well in intrasubject registration, cortical folds do not match well and may not effectively address the issues arising from the variability across a population (Hellier et al. 2003). Alternatively, cortical surfaces registration allows anatomically meaningful features to constrain the transformations (Tosun et al. 2004), which improves the reliability of the registration and rather favors the alignment of functional regions. Although variability in high level foldings, gyri and sulci, exists, the cortical shapes tend to present similar patterns at coarse levels (Tosun, Rettman, and Prince 2004). A good matching may be consequently found by the alignment of the lobes and major folding patterns at a coarse level across the brains, thereby registering cortical surfaces instead of volumetric data.

Reliable cortical surface registration for statistical comparisons raises different 
methodological questions which constrain any pre-processing step. The main issues are related with i) the preservation of gyri and sulci and with ii) the topology correction. Firstly, since the folding patterns correlate well with functional and anatomical regions between individuals (Fischl et al. 2008) a reliable detection of gyri and sulci during the segmentation step is required. Various methods have been proposed: Hutton (Hutton et al. 2008) used a layering method in the voxel space based on mathematical morphology to detect deep sulci. In (Acosta et al., 2008), a method is proposed to improve sulci detection by using a distance based cost function from WM in a post processing step, but no verification of topology is done. In (Rueda et al. 2010) the approach is improved by introducing topology constraints but also in a post-processing step, obtaining both reliable pure tissue and partial volume images segmentations. In (Cardoso et al. 2011) an explicit model of partial volume classes is introduced within the segmentation step and a locally varying MRF-based model is used to locally modify the priors for enhancement of gyri, but the topology is not guaranteed yet. Secondly, the GM can be considered as a folded sheet built upon the WM and it is often assumed that if the midline hemispheric connections were artificially removed, the cortex would have the topology of a hollow sphere (neither handles nor tunnels). This assumption must be preserved throughout all the processing pipeline. Topological operators and constraints have to be used to correct and achieve accurate cortical tissue representations either in the voxel space during the segmentation or in the surface representations (Ségonne and Fischl 2007), (Ségonne 2008), (Bazin and Pham 2005), (Han et al. 2002), (Kriegeskorte and Goebel 2001). The main drawback of correcting topology in the voxel space lies in the modifications introduced by adding or removing voxels compared to the size of the structures, besides the non unicity of the handle definition. Some other approaches used a combined voxel-based strategy (Jaume, Rondao, and Macq 2005), (Zhou, Ju, and $\mathrm{Hu} 2007$ ) and, as we mentioned before, other operates directly with the surfaces (CLASP (J. S Kim et al. 2005), BrainVISA (J-F. Mangin et al. 1995), so as Freesurfer (A.M. Dale, B. Fischl, and M.I. Sereno 1999), (B. Fischl, M. I Sereno, and A. M Dale 1999), (B. Fischl and A. M Dale 2000)). FreeSurfer incorporates mechanisms to prevent selfintersection of surfaces or topology correction, additionally imposing smoothness constraints, but at expense of computational cost. 
The goal of topology correction in surface-based methods is the elimination of defects appearing in the mesh such as tunnels and handles after the mesh generation (B. Fischl, M. I Sereno, and A. M Dale 1999), (Florent Ségonne and Bruce Fischl 2007), (Jaume, Rondao, and Macq 2005), (Zhou, Ju, and Hu 2007), (Hong et al. 2006). Some of them inflate the surface to detect the topological incoherences (B. Fischl, M. I Sereno, and A. M Dale 1999), (Florent Ségonne and Bruce Fischl 2007). Nevertheless, the optimization step for spherical inflating is non-deterministic and its complexity cannot be evaluated. Moreover, the corrections are not optimal from the topological point of view as the surfaces are not corrected according to the shortest loops that corresponds to the handles and tunnels. In this paper we addressed the issue of topology correction with a method based on the detection and optimal cutting of nonseparating loops in the mesh.

Concerning the surface based non-rigid registration, some of the previously proposed approaches have used a parametric representation on a sphere, obtained either through iterative relaxation like in Freesurfer (B. Fischl, M. I Sereno, and A. M Dale 1999) or conformal mapping (Angenent et al. 1999), (Gu et al. 2004). In the spherical domain, the registration strategy relies on features such as sulcal landmarks or mean curvature, or convexity (Yeo et al. 2010), (Yeo et al. 2008), (Ziolko et al 2006). In those approaches the global shape information is not explicit and the registration is dependent on the selected features. Shi et al. (Shi et al. 2007) proposed the computation of a direct map from the source to the target constrained by the sulcal landmark curves avoiding any parameterization. It assumes nevertheless that the sulcal landmarks curves are known and can be matched, which is not always the case. An intermediate unfolded (inflated) representation of the brain provides an alternative to expose hidden sulci and to simplify the geometry for cortical mapping preserving its global shape at a coarse level such as the main lobes and folds. Known as partially flattened surfaces (PFS) (Drury et al. 1996) , (B. Fischl, M. I Sereno, and A. M Dale 1999), (Pons, Keriven, and Faugeras 2004) they can also provide an intermediate step to further conformal mapping (Tosun, Rettman, and Prince 2004). In a previous work we compared different methods for obtaining PFS in terms of 
their ability to preserve areas and angles (Bonner et al, 2009). Compared to the original brain surface representations, the PFS simplifies the determination of shape correspondences between subjects while avoiding the spherical mapping procedure (Eckstein et al. 2007). A meaningful descriptor of shape in terms of brain anatomy may be used, thereby, as a similarity criterion.

In this paper we present a new cortical surface processing pipeline for vertex-wise interindividual analysis. We provide a comprehensive approach which performs the surface cortical mapping steps through MRI segmentation, surface generation, topology correction, inflation and surface matching, yielding a representation of a whole population in a common space. The matching between individuals' flattened surfaces (PFS) is based on the highly discriminative scale-invariant properties of the shape context (SC) (S. Belongie and J. Malik 2000), previously explored in (Acosta et al., 2010). The similarity between points is a combination of shape context and the information of sulci automatically extracted during an inflation step (sulci depth). The interpolation is obtained with the Thin Plate Spline (TPS) (Bookstein 1989). The major contribution of the shape context lies in the global shape characterization at a local level for each single point. Our method produces an anatomically meaningful and scale-invariant matching between the lobes and the major folding patterns.

The $\mathrm{C}++$ code and binaries for segmentation, cortical thickness estimation (CTE) proposed in (Acosta et al, 2009) and the topology correction are fully available at our CSIRO software website ${ }^{3}$. The proposed pipeline, allows also the comparison of different imaging biomarkers for other neurodegenerative diseases. In the remainder of the paper we explain the following steps: generation of genus zero surfaces (Section 2.1), PFS (Section 2.2) and a description of the shape context (Section 2.3.1). The validation experiments using real MRI are then described and a clinical application in an Alzheimer's disease study is also presented (Section 3.4) where we tested the ability of cortical thickness as a biomarker for atrophy to compare two populations.

3 http://research.ict.csiro.au/software/milxview 
[ Figure 1 : Overall process for cortical mapping] 


\section{Materials and Methods}

The proposed method consists of several stages as summarized in Fig. 1: Firstly, 3D T1weighted MR images are classified into GM, WM and CSF in their original space using an expectation maximisation segmentation (EMS) algorithm (Van Leemput et al. 1999), as described in (Bourgeat et al. 2009). The EMS computes probability maps for each tissue type, which are then discretized by assigning each voxel to its most likely tissue type. This scheme has been improved in (Bourgeat et al. 2009) allowing the use of multiple ( $n$ ) atlases for subsequent segmentations and parcellations, in order to improve the reliability of the priors. Thus, each individual scan is segmented $n$ times, once for each of the $n$ atlases. The resulting $n$ segmentations per patient are then combined using a voting scheme to provide consensus segmentation according to (Aljabar et al. 2009). Similarly, for each patient, the $n$ propagated Automated Anatomical Labeling (AAL) (Tzourio-Mazoyer et al. 2002) and the Internet Brain Segmentation Repository (IBSR) labeling ${ }^{4}$, are also combined using the same voting scheme. This step ensures that outliers resulting from poor spatial normalization are excluded, and only regions of high confidence are preserved. Secondly, based on these pure tissue segmentations, a further maximum a posteriori classification of voxels into pure tissues WM, GM and CSF and mixed tissues WM/GM and GM/CSF along the previously computed GM interface is performed, which results in a GM partial-volume coefficients (GMPVC) image used for further cortical thickness estimation (Acosta et al. 2009). Topology-constraints are introduced in the voxel classification assuming that the GM is a continuous layer covering a WM, homotopic to a filled sphere as presented previously in (Rueda et al. 2009). A topology preserving dilation of the WM over the GM improves the robustness of delineation of mixed GM/CSF voxels in deep sulci. The propagated IBSR labeling is then used as a mask to separate left and right hemispheres, after which the generation of 3D polygon meshes representing the WM/GM interface is performed with the marching cubes algorithm (Lorensen and Cline 1987). Tunnels and handles resulting from the meshing are removed with a method based on the detection and correction of nonseparating loops (Fig. 3). In order to reduce the surface convolution and the inter-individual 
variability, the surfaces are then inflated to a similar shape (Fig. 4). The inflated surfaces, referred to as PFS, are finally elastically registered towards the common template using the shape context-based non rigid registration (Fig. 5). The registration imposes a standardized coordinate system on both surfaces, allowing the vertex-wise comparison of the whole population. In this paper, we detail and validate the steps 5 to 7 in Fig. 1 and illustrate the results with an example of clinical application in which the cortical thickness was computed using our voxel-based approach (Acosta et al. 2009) propagated to the registered meshes. We compared the differences in cortical thickness between a population of Alzheimer's disease (AD) patients and a control group of healthy elderly individuals (HC).

\subsection{Correction of topology and generation of genus zero surfaces}

After the surfaces are generated by the Marching Cubes algorithm (Lorensen and Cline 1987) the topology is corrected to meet the assumed topological properties of the brain, homeomorphic to a sphere. Handles and tunnels appear as topological defects to be removed. To this end, we implemented an iterative topological correction inspired by (Erickson and HardPeled 2002) allowing to remove one by one the unwanted handles and tunnels.

Let $g$ be the genus of the surface $M$, defined as the number of handles and tunnels in M. $g$ can be easily computed using the Euler's characteristic $\chi(\mathrm{M})$ as $g=\frac{2-\chi(\mathrm{M})}{2}$, where $\chi(\mathrm{M})=V(\mathrm{M})-E(\mathrm{M})+T(\mathrm{M})$ and $V(\mathrm{M}), E(\mathrm{M})$ and $T(\mathrm{M})$ are respectively the number of vertices, edges and triangles in $\mathrm{M}$.

The algorithm (Algorithm 1) iteratively corrects the topology, reducing the genus of $M$ by one at each step. After $g$ corrections, filling the tunnels and cutting the handles, the new surface is homeomorphic to a sphere.

Observe that in a first step the global algorithm is applied aimed at closing all the

4

http://www.cma.mgh.harvard.edu/ibsr 
possible boundaries of the original surface. The removal of a handle or a tunnel is performed by first computing an approximation of the shortest non-separating loop at each topological defect (detailed below and summarized in the Algorithm 2). A non-separating loop is a connected path that does not divide the surface into two connected components. The surface $\mathrm{M}$ is cut along the $n$ edges of this identified loop, therefore adding $n$ edges and $n$ vertices to the mesh. At this step, two new boundaries are created but the Euler's characteristic is not modified. The final correction is performed by sealing the created boundaries with small discs, thus adding $n$ edges $n$ triangles and one vertex (we add an umbrella). This increases the Euler's characteristic by one, globally reducing the genus by one.

Algorithm 2 describes the computation of the shortest non-separating loop. We first compute a set of basepoints $\mathrm{B}$ by propagating a wavefront from a randomly selected vertex (a seed point) in $\mathrm{M}$. B is defined as the set of vertices where the two wavefront boundaries meet (Figure 2a). The complement of $\mathrm{B}$ in $\mathrm{M}$ is a disc, implying that any non-separating loop on $\mathrm{M}$ contains at least one point of $\mathrm{B}$. Figure $2 \mathrm{~b}$ is an example of cut locus on a 2-genus surface.

Using this property we compute the non-separating loops from all the vertices contained in the path B. This has been proved by (Erickson and Hard-Peled 2002) as a good approximation of the shortest non-separating loop in $M$. Unlike the original approach, in which each point of $B$ is used to compute a non-separating loop, we perform this computation using propagations from a non-trivial set of points, which speeds-up the computation. As illustrated in Figure 2b, the cut locus B is subdivided into a finite (and small) number of paths, like $\mathrm{S}_{\mathrm{B}}$ in Figure 2c. For each of these paths, we compute a wavefront from the contained points into the complement of $\mathrm{B}$ in $\mathrm{M}$. When the two boundaries of this wavefront meet, an associated loop is computed from the junction point going back to the initial wavefront seed. This, at each side of the junction. The non-separating property of this loop is verified by checking the number of connected components of the complement. Selecting a good approximation of the shortest non-separating loop at each step guarantees a minimal structural 
modification of the surface $M$. Figure 3 shows topological corrections applied to a tunnel (figure 3a, 3b) and to two handles (figure 3c, 3d).

[Figure 2]

[Figure 3]

\subsection{Partially flattened surfaces}

A number of methods have been proposed in the literature for unfolding or flattening cortical surfaces (Drury et al. 1996), (B. Fischl, M. I Sereno, and A. M Dale 1999) , (Pons, Keriven, and Faugeras 2004). In order for a flattening to be useful, it must preserve local and global metrics such as triangle angles and areas. For our purposes we implemented a method based upon CARET (Drury et al. 1996), (Tosun, Rettman, and Prince 2004). This method was compared with the method implemented in Freesurfer (Bonner et al, 2009). In our implementation, a cortical surface is iteratively deformed at each vertex according to $m_{i}^{t+1}=(1-\lambda) m_{i}^{t}+\lambda \bar{m}_{i}^{t}$, where $m_{i}^{t}$ is the position of vertex $i$ for iteration $t, \lambda$ is a scalar in the range $[0,1]$ and $\bar{m}_{i}^{t}$ represents the average vertex position of $m_{i}^{t}$, given by

$$
\bar{m}_{i}^{t}=\frac{1}{\sum S_{j}^{t}} \sum_{j \in N_{i}} S_{j}^{t} c_{j}^{t}
$$

where $N_{i}$ is the set of all triangles containing $m_{i}, c_{j}$ is the center of triangle $j$ and $S_{j}$ is its area. Typically a factor of 0.9 is chosen for $\lambda$, giving more weigh to the averaged vertex position. This process moves all mesh vertices towards the weighted average of the centers of their surrounding triangles. The deformation progresses until the global mean curvature $\bar{k}$ drops below a predefined threshold (Tosun, Rettman, and Prince 2004) or in our case using a predefined number of iterations (we used typically 400), obtaining similar shapes between moving and fixed surfaces. Fig. 4 (Top) shows the different steps in the evolution of the PFS with the sulcal depth maps computed at each step. The color coded sulcal depth map allows 
the visual localization of the main folding patterns. The sulcal depth map is computed as the cumulative path length at each point $i$ as

$$
\operatorname{depth}_{i}=\sum_{t=[1, \ldots, N]}\left[m_{i}^{t+1}-m_{i}^{t}\right]
$$

where $N$ is the number of iterations. Fig 4 Bottom depicts the comparison of Area distortion after inflation using CARET and Freesurfer.

[Figure 4]

\subsection{Surface-based non-rigid registration}

Surface based non-rigid registration methods face several challenges including: $i)$ the choice of similarity criterion and ii) the matching and global optimization procedure (Audette, Ferrie, and Peters 2000). The first one refers to the type of information extracted from the 3D surface, namely the description of local or global shape to represent the similarity. The latter challenge concerns the exploitation of the similarity information to find the best matching between the two surfaces. In this context, the goal of the registration is to determine the transformation such that for a finite set of control points, any control point of a moving surface $\mathrm{M}$, is mapped onto the corresponding control point of a fixed surface $M$.

\section{[Figure 5]}

Fig. 5 illustrates the method used to register the moving $\mathrm{M}=\left\{\mathrm{m}_{i}\right\}_{i=1 \ldots p}$ and fixed $\mathrm{F}=\left\{\mathrm{f}_{j}\right\}_{j=1 \ldots q}$ PFS surfaces, respectively. F may be a template towards which we register the whole population. Let $\mathrm{M}_{\mathrm{s}}=\left\{\mathrm{m}_{k}\right\}_{k=1 \ldots u}$ and $\mathrm{F}=\left\{\mathrm{f}_{l}\right\}_{l=1 \ldots v}$ be the corresponding simplified surfaces, represented in two subsets of characteristic points (control points) of $\mathrm{M}$ and $\mathrm{F}$, such that $\mathrm{M}_{\mathrm{s}} \subseteq \mathrm{M}, \mathrm{F}_{\mathrm{s}} \subseteq \mathrm{F}$. After establishing bijective correspondences between the sets $\mathrm{M}_{\mathrm{s}}$ and 
$\mathrm{F}_{\mathrm{s}}$, exploiting a given similarity metric (in this paper the shape context), the mapping is computed as a set of transformations $\mathrm{T}=\left\{\mathrm{T}_{k}\right\}_{k=1 \ldots u}\left(\mathrm{~T}_{\mathrm{k}} \in \mathrm{R}^{3} \rightarrow \mathrm{R}^{3}\right)$ such that $\mathrm{f}_{k}=\mathrm{T}_{k} \circ \mathrm{m}_{\mathrm{k}}, \forall \mathrm{m}_{\mathrm{k}}$.

\subsubsection{Shape context}

The shape context is a shape descriptor that, for a single surface, captures the distribution of points over relative positions of the global shape points. This characterization is invariant to scale and rigid transformations, naturally leading to a highly discriminative and robust score for measuring shape similarities. The shape context was first introduced in (S. Belongie and J. Malik 2000) within the 2D pattern recognition field, and aimed to match point clouds representing similar patterns. Further modifications to the method appeared in (G. Mori, S. Belongie, and J. Malik 2005) and 3D matching of features have been extended to work with thoracic images (Urschler and Bischof 2004). A first implementation of 3D Shape Context for brain registration was presented in (Acosta et al, 2010).

Let $\mathrm{M}_{\mathrm{s}}$ and $\mathrm{F}_{\mathrm{s}}$ the two simplified PFS surfaces corresponding to the meshes $\mathrm{M}$ and $\mathrm{F}$ to be registered. $\mathrm{M}_{\mathrm{s}}$ and $\mathrm{F}_{\mathrm{s}}$ constitute the $\mathrm{u}$ control points (typically $\mathrm{u}=3000$ points) of the moving and fixed surfaces, respectively, to be matched and for which the shape context is computed (Fig. 5). Here, they are obtained using an algorithm that iteratively contracts vertex pairs while minimizing geometric errors (Garland and Heckbert 1997). For a given point $\left\{\mathrm{m}_{k}\right\}_{k=1 \ldots u} \in \mathrm{M}_{\mathrm{s}}$, its shape context is the 3D histogram of the relative 3D polar coordinates $(\vec{r}, \theta, \phi)$ of the remaining $u-1$ points. As in (Belongie et al 2002), in order to be more sensitive to nearby points, we use a log-polar coordinate system. In our case we build a 3D histogram with $R_{\vec{r}}$ equally spaced log-radius bins and $u_{\theta}$ and $v_{\varphi}$ equally spaced angle bins. Since the intracranial volume may considerably differ across a population, an additional normalization of the shape context is performed to obtain scale invariance. To this end, all the radial distances $\operatorname{dist}\left(\mathrm{m}_{\mathrm{k}}, \mathrm{m}_{1}\right)_{k, l=1 \ldots u},\{k \neq l\}$, are normalized by the mean distance between all the point pairs in the shape: 


$$
\frac{1}{u} \sum_{\substack{k, l \\ k \neq l}} \operatorname{dist}\left(\mathrm{m}_{\mathrm{k}}, \mathrm{m}_{1}\right)
$$

\subsubsection{Cost function and matching}

The shape context cost $C_{i, j}^{S C}$ of matching the point $\mathrm{m}_{\mathrm{i}}$ from the simplified surface $\mathrm{M}_{\mathrm{s}}$ with the point $\mathrm{f}_{\mathrm{j}}$ from $\mathrm{F}_{\mathrm{s}}$ is given by the $\chi^{2}$ statistic as

$$
C_{i, j}^{S C}=\frac{1}{2} \sum_{k=1}^{K} \frac{\left[h_{i}(k)-g_{j}(k)\right]^{2}}{h_{i}(k)+g_{j}(k)}
$$

where $h_{i}(k)$ and $g_{j}(k)$ are the histograms (shape context) at the points $i$ and $j$ of the moving $\mathrm{M}_{\mathrm{s}}$ and fixed $\mathrm{F}_{\mathrm{s}}$ shapes, respectively, and $K$ is the total number of bins.

In addition to the description given by the shape context, we use the sulcal depth map computed at each point according to equation (2) to enrich the matching between the points with local information about sulci and gyri. Thus, in a second iteration we recompute the depth map cost function $C_{i, j}^{S D}$ as :

$$
C_{i, j}^{S D}=\frac{\left|s_{j}-r_{j}\right|}{N}
$$

Where $s_{i}$ and $r_{j}$ are the sulcal depths at points $i$ and $j$ of the moving $\mathrm{M}_{\mathrm{s}}$ and fixed $\mathrm{F}_{\mathrm{s}}$ shapes respectively. Both are normalized by the brain size. $N$ is the highest difference between the floating and the fixed normalized sulcal depths; this enables the comparison between the sulcal depth and the shape context cost. The global cost function becomes then:

$$
C_{i, j}=\alpha C_{i, j}^{S C}+\beta C_{i, j}^{S D}
$$

where $\beta=1-\alpha$, and in practical terms $\alpha=0.8$, resulting in the best trade-off for a good matching of the folds. Given the individual costs $C_{k, l} \in[0, \ldots, 1]$ between all pairs of points, the 
next step is to find the perfect matching by minimizing the total cost of the bijective correspondences $\mathrm{H}=\sum \mathrm{C}$. This is done within a one to one point matching step with the Hungarian algorithm (Kuhn 1955). After the correspondences are found, the set of transformations $\mathrm{T}=\left\{\mathrm{T}_{\mathrm{k}}\right\}_{k=1, \ldots, u}$ for each point are computed. Finally, a transformation using a thin plate spline model (TPS) (Bookstein 1989) calculated from the control points, achieves the interpolation of the corresponding moving shape $\mathrm{M}$ onto $\mathrm{F}$. In our implementation, the number of points in $\mathrm{M}_{\mathrm{s}}$ is lower than in $\mathrm{F}_{\mathrm{s}}$ to avoid miscorrespondences or topological errors due to crossing points between $\mathrm{M}_{\mathrm{s}}$ and $\mathrm{F}_{\mathrm{s}}$. Fig. 6 shows an example of labeling, propagated from a template to a single individual's surface after registration of the corresponding PFS. 


\section{Experiments and results}

This section describes the experiments performed to validate each step of the pipeline using real data and shows with an example the application on clinical data for quantifying atrophy in Alzheimer's Disease using cortical thickness. We also compared the results with Freesurfer (A.M. Dale, B. Fischl, and M.I. Sereno 1999), (B. Fischl, M. I Sereno, and A. M Dale 1999), (B. Fischl and A. M Dale 2000). All the methods were implemented in C++, incorporated in a plugin called milxCTE devised to compute the cortical thickness as described in (Acosta et al. 2009), and is part of our downloadable software platform milxView ${ }^{5}$, and TAGLUT (Topological And Geometrical Library - a Useful Toolkit) ${ }^{6}$ (Favreau 2009), which provides the topological and geometrical tools for genus zero mesh generation. Milxview utilises the open source ITK ${ }^{7}$ and VTK ${ }^{8}$ libraries.

\subsection{Data}

\subsubsection{Cross sectional MR scans}

From the Open Access Series of Imaging Studies (OASIS) database (Marcus et al. 2007) ${ }^{9}$, we randomly selected 30 young healthy individuals. The scans were T1-weighted Magnetization Prepared RApid Gradient Echo (MP-RAGE) in sagittal orientation with isotropic $1 \mathrm{~mm}^{3}$ resolution (256x256x128 pixels). For the first set of experiments, inflation and registration parts, the segmentations of the WM/GM interface were computed using Freesurfer. Thus, for each individual we obtained separated surface representations for left and right hemispheres. The SC was used to register the surfaces to a common template, which was obtained after segmentation of the Colin atlas(D.L. Collins et al. 1998) with Freesurfer. The resulting meshes contained an average of 300000 vertices.

\footnotetext{
5 http://aehrc.com/biomedical_imaging/milx.html

6 http://www.jmfavreau.info/?q=en/taglut

7 http://www.itk.org/

$8 \quad$ http://www.vtk.org/

$9 \quad$ www.oasis-brains.org
} 


\subsubsection{Clinical data in Alzheimer's disease: the AIBL study}

For the application of the proposed method to the study of Alzheimer's disease, MR images from $A D$ patients, individuals with mild cognitive impairment $(\mathrm{MCl})$, and $\mathrm{HC}$ were included. They were randomly selected from the individuals enrolled in the Alzheimer Imaging Biomarkers and Lifestyle longitudinal (AIBL) study (Kathryn A Ellis et al. 2009). The AD participants met NINCDS-ADRDA criteria for probable AD (McKhann et al., 1984 ) while all the $\mathrm{MCl}$ subjects met the Petersen criteria (Petersen et al., 1999) of subjective and objective cognitive difficulties, in the absence of dementia or significant functional loss. The remaining participants in the study were healthy elderly volunteers. All patients were recruited from the Austin Health Memory Disorders and Neurobehavioural Clinics. All of the subjects were scanned with a standardized protocol. Thus, sagittal T1 weighted MR images were acquired using a standard 3D MPRAGE sequence at 3T, with in-plane resolution $1 \times 1 \mathrm{~mm}$, slice thickness $1.2 \mathrm{~mm}, \mathrm{TR} / \mathrm{TE} / \mathrm{T} 1=2300 / 2.98 / 900$, flip angle $9^{\circ}$ and field of view of $240 \times 256$ voxels and 160 slices. The protocol of acquisition and demographics are fully detailed in (P. Bourgeat et al. 2009). The SC was used to register the surfaces to a template, which was obtained as an average of a subsample (20 individuals) randomly selected from the whole population.

\subsection{Partially inflated surfaces}

Using the 30 young healthy individuals randomly selected from the OASIS database (Marcus et al. 2007), we first compared the results of the inflation step with Freesurfer. In this experiment, segmentations of the WM/GM interface were computed using Freesurfer. Thus, for each individual we obtained separated surface representations for left and right hemispheres. We evaluated the computation of PFS separately in terms of area preservation. A thorough comparison of different methods using local and global area, angle and length distortions was presented in (Bonner et al. 2009). In this paper we only report the measured local metric distortion in the 1-neighbourhood surrounding each mesh vertex. The local area distortion was defined for each vertex $i$ as 


$$
\hat{M}_{A}(i)=\frac{\sum_{t \in \widehat{T}} A(t)}{\sum_{t \in \widehat{T}} A^{0}(t)}
$$

and the length distortion as

$$
\hat{M}_{D}(i)=\frac{\sum_{j \in \hat{N}_{1}} d_{i j}}{\sum_{j \in \hat{N}_{1}} d_{i j}^{0}}
$$

where $\widetilde{T}$ is the set of triangles containing vertex $i, \widetilde{N}_{1}$ is the set vertices in the 1 neighbourhood of $i, A(t)$ is the area of triangle $t$ and $A^{0}(t)$ is the area of triangle $t$ on the original mesh. This is a measure of the average absolute change in area per triangle on the mesh. $d_{i n}^{0}$ and $d_{i n}$ are the distances between vertex $i$ and $n$ on the original and modified surfaces, respectively. In our experiments, the implemented method for computation of PFS has been proven (Bonner et al, 2009) to perform better than FreeSurfer (B. Fischl, M. I Sereno, and A. M Dale 1999). Fig 4 Bottom depicts a comparison of the local area distortion after inflation using CARET and Freesurfer. Averaging over the 30 individual brains surfaces, it produced substantially fewer area and distance distortions. $45.89 \%$ of vertex distortions ranged within $[0.8,1.2]$ (1.0 represents no distortion), compared to $34.83 \%$ for FreeSurfer. Table 1 shows the average portion of vertices within this range for each method, computed for all 30 subjects in the sample space per lobe. Overall, the distance metric was distorted nearly equally by both CARET and FreeSurfer $(23.03 \pm 0.86 \%$ for CARET vs $23.11 \pm 1.05 \%$ for FreeSurfer) whereas the area was distorted by $32.7 \pm 1.42 \%$ using CARET vs $39.09 \pm 1.71$ with Freesurfer.

\subsection{Shape context-based non rigid registration}

The overall method was used to register the obtained surfaces to a common template. For the experiments described using OASIS the template was obtained as a surface representation of the Colin atlas (D.L. Collins et al. 1998). This template was obtained with 
Freesurfer applying the segmentation pipeline to the initial Colin's T1-W MRI. For the experiments using clinical data a different template was obtained as an average surface representation of the population being studied.

In the first experiment using the OASIS database, we investigated the effect of the number of control points $(u)$ employed to represent the simplified surfaces $M_{s}$ and $F_{s}$ on the registration quality. Mean absolute (MAD) and Hausdorff (HD) distances (Gerig, Jomier, and Chakos 2001) between moving and target surfaces were used to assess the accuracy of our method to register two PFSs. Table 2 presents a summary of these results. By Increasing $u$ from 500 to 1000 control points the accuracy accuracy in terms of mean absolute distance (MAD) was improved by 33\% ( $p<0.0001)$ and by 32\% ( $p<0.0001)$ when varying from 1000 to 2000 points for the left hemisphere and 23\% $(p<0.0001)$ and $27 \%(p<0.01)$ respectively for the right hemisphere. As expected, surface alignment is better when using more control points. A trade-off between computational requirements and accuracy exists and must be defined depending on the application.

\subsubsection{Overlap with Freesurfer labeling}

In this experiment, we compared the localization of Freesurfer labeled regions (obtained as described in (Desikan et al. 2006)) after registration to the same template (Fig. 6). The Jaccard coefficient measured as the relation between the intersection and union of areas of labelled regions was used as the similarity metric. Overall, our method is comparable with the registration provided with Freesurfer. Averaging for the 30 individuals, in 6 regions of the left hemisphere and 6 of the right hemisphere the overlap (Jaccard) obtained with our method was higher than with Freesurfer. In other regions the accuracy was lower than with Freesurfer but in most of them these differences they were not statistically significant, except for the entorhinal cortex, middle temporal, pars triangularis and transverse temporal (Desikan et al. 2006) in both hemispheres. Table 3 shows some examples of the jaccard coefficient averaged on the 30 individuals. The worst values were obtained in the smallest regions, where the overlap is highly affected by misregistration errors. Conversely in larger areas the overlap was fairly good, and 
the main folds were well aligned. It is important to notice that the statistical analysis will be performed later at a voxel basis. Fig. 6 illustrates an example of propagated labels between an individual and the template. It can be observed that although there is a high interindividual variability, the regions are well transferred through all the foldings representing different anatomical regions.

[Figure 6]

\subsection{Clinical data: AIBL study}

Following the procedure detailed in Section 2, consensus segmentations were obtained as described in (Bourgeat et al. 2009) using nine atlases for initialization. Subsequently, cortical thickness maps were computed using our voxel-based approach (Acosta et al. 2009). The individuals' Automated anatomical labeling (AAL) (Tzourio-Mazoyer N, et al 2002) and cortical thickness were propagated to the resulting mesh and then to the average template after the SC registration.

As an example to describe the matching of similar regions of the brain, we randomly selected 26 individuals. Their consensus AAL labelings, obtained in the previous step, were propagated towards the template applying the whole pipeline. The labelings were then combined in a voting scheme to obtain a final AAL labeling in the template space (Fig. 9). After the registration, consistency in the coincidence of the major folding patterns was obtained across the individuals.

\subsubsection{Cortical thickness comparison with Freesurfer}

Cortical thickness maps from 50 individuals obtained with FS and milxCTE (Acosta et al. 2009) were compared. After registration and mapping to the common template, the thickness maps were regionally compared. Cortical thickness was comparable in all the regions. Similar patterns for thickness in gyri and sulci were obtained. Overall, a strong correlation between the results using both methods FS and milxCTE was found. In average, for all the regions the absolute computed difference was $0.23 \mathrm{~mm}(r=0.63)$ for the left hemisphere and $0.17 \mathrm{~mm}$ ( $r=0.65)$ for the right. Table 4 shows the results for some of the regions, left and right 
hemispheres.

[Figure 7]

[Figure 8]

Figure 7 shows the results of the correlations $(r)$ for all the regions. Similar results were obtained in both hemispheres. Some examples of the individuals' thickness computed with milxCTE and FS are depicted in Fig. 8. As suggested in (Klauschen et al. 2009) Fresurfer tends to underestimate gray matter, therefore the computed thickness with our method tends to be higher. Although the slope on Fig. 8 is leaning towards the higher values of CTE, the correlations are still high as shown in table 4 . In milxCTE the thickness estimation is entirely voxel-based with a sub-voxel initialization using partial volume maps (Acosta et al. 2009). This makes it very fast and accurate whereas FS is based on meshes deformations which may be computationally expensive. Not surprisingly, larger regions: Supramarginal (2400 vertices), Inferior parietal (4200), superior temporal (2600), superior frontal (4500) presented the highest correlations as opposed to smaller regions such as rostral anterior cingulate (400), frontal pole (60) or caudal anterior cingulate (813) where small overlap differences may affect the measure.

\subsubsection{Study of cortical thickness in Alzheimer's disease}

We also investigated the ability of our method to detect cortical thickness differences between 81 healthy elderly individuals $\mathrm{HC}$, and $32 \mathrm{AD}$ patients. Fig. 9 shows an example of cortical thickness maps from two individuals, one from each group, propagated to the common template.

[Figure 9]

[Figure 10]

After applying the whole pipeline to all the individuals, vertex-wise t-tests were performed between NC-AD to identify regions where a significant atrophy existed. The cortical thickness values were corrected for age and False Discovery Rate (FDR) corrected p-values were 
obtained ( $5 \%$ threshold). In our experiments, the analysis was performed using the thickness maps with a Laplacian smoothing ( $5 \mathrm{~mm}$ Full Width at half of the Maximum-FWHM) over the scalar cortical thickness maps to reduce the effects of discontinuities. The obtained differences between the two groups are illustrated in Fig. 10 and demonstrated qualitatively the consistency of the results. The results show significant differences between the two groups, with lowest thickness for the AD group with both methods. Significant differences were found in the hippocampus, parahippocampus, cingulate and in the temporal and frontal lobes between the two groups. In average, the absolute differences in cortical thickness for some regions were higher by using our method, as it can be seen in Figure 10 and in Table 4. These results corroborate what has been previously reported in the literature for Alzheimer's disease (Lerch and A. C Evans 2005), (Querbes et al. 2009). 


\section{Conclusion}

In this paper we presented a new cortical surface processing pipeline, milxCTE, for accurate statistical regional analysis. It offers an alternative to traditional methods using surfac representations of the brain such as Freesurfer. It involved several steps, namely MRI segmentation, surface generation, topology correction, inflation and surface matching, yielding a representation of a whole population in a common space. In addition to the shape context which provides a global description at a local level, we included additional local landmarks, such as the sulcal depth map, to iteratively adjust the registration, yielding a meaningful matching between lobes. An update of the binary distribution of milxCTE and milxView is available ${ }^{10}$.

We demonstrated the validity of the method for use in clinical studies, by evaluating each step separately on real data, and then comparing the overall technique against Freesurfer. This comparison showed that the labeling of major folding patterns is preserved and the cortical thickness computed by using our method is regionally consistent. In average, with Freesurfer the obtained values of cortical thickness were lower than with milxCTE for single individuals. This is not a surprising finding as it has been previously shown and reported in larger studies. Our method performs the computation of cortical thickness in the voxel domain, which is very fast. Additionally, it uses advantageously the partial volume information to initialize at a subvoxel level, thereby yielding a very accurate result. However, a good correlation between both measures were found and different experiments comparing averaged cortical thickness between two populations allowed to measure the relative differences of atrophy. This preliminary study on clinical data showed regional differences between healthy elderly individuals and Alzheimer's disease patients. The most significant atrophy was measured in the temporal lobe, which is consistent with the published literature.

We intend to perform longitudinal clinical studies on Alzheimer's disease and other neurological disorders. As similar results were found in regional comparisons with Freesurfer, our method represents an alternative for the study of cortical thickness estimation across a population.

As the method allows the mapping of a whole population in a common space, in future 
work we will perform multi-feature comparison of additional imaging biomarkers for Alzheimer's disease. Thus, not only cortical thickness is compared but also other features from different modalities such as sulcal depth, WM/GM MRI contrast, GM volume, $\beta$-Amyloid burden or WM integrity in the same vertex-wise common space. 


\section{References}

Acosta O, Bourgeat P, Zuluaga MA, Fripp J, Salvado O, Ourselin S, and Alzheimer's Disease Neuroimaging Initiative. 2009. Automated voxel-based 3D cortical thickness measurement in a combined Lagrangian-Eulerian PDE approach using partial volume maps. Med Image Anal 13, no. 5 (October): 730-743.

Acosta O, Bourgeat P, Fripp J, Bonner E, Ourselin S and Salvado O, Automatic delineation of sulci and improved partial volume classification for accurate 3D voxel-based cortical thickness estimation from MR, Lecture Notes in Computer Science - MICCAI (2008), pp. 253-261.

Acosta, O.; Fripp, J.; Rueda, A.; Xiao, D.; Bonner, E.; Bourgeat, P. and Salvado, O. 3D shape context surface registration for cortical mapping Proc. IEEE Int Biomedical Imaging: From Nano to Macro Symp, 2010, pp. 1021-1024.

Aljabar P, Heckemann RA, Hammers A, Hajnal JV, and Rueckert D. 2009. Multi-atlas based segmentation of brain images: Atlas selection and its effect on accuracy. Neuroimage 46: 726-738.

Angenent S, Haker S, Tannenbaum A, and Kikinis R. 1999. On the Laplace-Beltrami operator and brain surface flattening. IEEE Transactions on Medical Imaging 18 (August): 700-711.

Ashburner J, and Friston KJ. 2000. Voxel-based morphometry-the methods. Neuroimage 11, no. 6 Pt 1 (June): 805-821.

Audette MA, Ferrie FP, and Peters TM. 2000. An algorithmic overview of surface registration techniques for medical imaging. Medical Image Analysis 4, no. 3: 201-217. doi:DOI: 10.1016/S1361-8415(00)00014-1.

Bazin PL, and Pham DL. 2005. Topology Correction Using Fast Marching Methods and Its Application to Brain Segmentation. In Medical Image Computing and Computer-Assisted Intervention 2005, 484 - 491. Lecture Notes in Computer Science. Springer Verlag.

Belongie S, and Malik J. 2000. Matching with shape contexts. Content-based Access of Image and Video Libraries, 2000. Proceedings. IEEE Workshop on: 20-26. doi:10.1109/IVL.2000.853834.

Belongie S, Malik J, and Puzicha J. 2002. Shape matching and object recognition using shape contexts. IEEE Transactions on Pattern Analysis and Machine Intelligence 24: 509-522.

Bonner E, Acosta O, Fripp J, and Salvado O. 2009. A quantitative comparison of three methods for inflating cortical meshes. In 2009 IEEE International Symposium on Biomedical Imaging: From Nano to Macro, 1338-1341. Boston, Massachusetts, USA: IEEE, June.

Bookstein FL. 1989. Principal warps: thin-plate splines and the decomposition of deformations. Pattern Analysis and Machine Intelligence, IEEE Transactions on 11, no. 6: 567-585.

Bourgeat $P$, Chételat G, Villemagne V, Fripp J, Raniga P, Pike K, Acosta O, et al. 2009. AB burden in the temporal neocortex is related to hippocampal atrophy in non-demented elderly. Neurology.

Cardoso, M. J., Clarkson, M. J., Ridgway, G. R., Modat, M., Fox, N. C., Ourselin, S., The Alzheimer's Disease Neuroimaging Initiative. 2011. LoAd: A locally adaptive cortical segmentation algorithm.. Neuroimage56 (3), 1386-1397

Chételat G, Desgranges B, Landeau1 B, Mézenge F, Poline JB, de la Sayette V, Viader F, 
Eustache F, and Baron JC. 2007. Direct voxel-based comparison between grey matter hypometabolism and atrophy in Alzheimer's disease. Brain 131, no. 1 (January): 60-71.

Collins DL, Zijdenbos AP, Kollokian V, Sled JG, Kabani NJ, Holmes CJ, and Evans AC. 1998. Design and Construction of a Realistic Digital Brain Phantom. IEEE Transactions on Medical Imaging 17, no. 3 (June): 463-468.

Dale AM, Fischl B, and Sereno MI. 1999. Cortical surface-based analysis I: Segmentation and surface reconstruction. Neurolmage 9, no. 2 (February): 179-194.

Desikan RS, Segonne F, Fischl B, Quinn BT, Dickerson BC, Blacker D, Buckner RL, et al. 2006. An automated labeling system for subdividing the human cerebral cortex on MRI scans into gyral based regions of interest. Neurolmage 31, no. 3: 968-980. doi:DOI: 10.1016/j.neuroimage.2006.01.021.

Dijkstra EW. 1959. A note on two problems in connexion with graphs. Numerische Mathematik 1, no. 1: 269-271. doi:10.1007/BF01386390.

Drury HA, Van Essen DC, Anderson CH, Lee CW, Coogan TA, and Lewis JW. 1996. Computerized Mappings of the Cerebral Cortex: A Multiresolution Flattening Method and a Surface-Based Coordinate System. Journal of Cognitive Neuroscience 8, no. 1: 128. doi:10.1162/jocn.1996.8.1.1.

Eckstein I, Joshi AA, Jay Kuo CC, Leahy RM, and Desbrun M. 2007. Generalized Surface Flows for Deformable Registration and Cortical Matching. In MICCAI (1), 692-700.

Ellis, Kathryn A, Bush Al, Darby D, De Fazio D, Foster J, Hudson P, Lautenschlager NT, et al. 2009. The Australian Imaging, Biomarkers and Lifestyle Study of ageing (AIBL): methodology and baseline characteristics of 1112 individuals recruited for a longitudinal study of Alzheimer's disease. International Psychogeriatrics 21, no. 4 (August): 672-687.

Erickson J, and Har-Peled S. 2002. Optimally cutting a surface into a disk. In Proceedings of the eighteenth annual symposium on Computational geometry (SCG '02), 244-253. New York, NY, USA. doi:http://doi.acm.org/10.1145/513400.513430.

Favreau, JM. 2009. TAGLUT: Topological And Geometrical Library - a Useful Toolkit.

Fischl B, and Dale AM. 2000. Measuring the thickness of the human cerebral cortex from magnetic resonance images. Proceedings of the National Academy of Sciences of the United States of America 97, no. 20 (September): 11050-11055.

Fischl B, Sereno MI, and Dale AM. 1999. Cortical Surface-Based Analysis II: Inflation, Flattening, and a Surface-Based Coordinate System. Neurolmage: 195-207.

Fischl B, Rajendran N, Busa E, Augustinack J, Hinds O, Yeo B. T. T., Mohlberg H, Amunts K. and Zilles K, Cortical folding patterns and predicting cytoarchictecture, Cerebr. Cortex, 2008vol. 18, no. 8, pp. 1973-1980.

Ségonne F, Pacheco J, and Fischl B. 2007. Geometrically Accurate Topology-Correction of Cortical Surfaces Using Nonseparating Loops. IEEE Transactions on Medical Imaging 26, no. 4: 518-529.

Garland M, and Heckbert PS. 1997. Surface simplification using quadric error metrics. In SIGGRAPH '97: Proceedings of the 24th annual conference on Computer graphics and interactive techniques, 209-216. New York, NY, USA: ACM Press/Addison-Wesley Publishing Co. doi:http://doi.acm.org/10.1145/258734.258849.

Gerig G, Jomier M, and Chakos A. 2001. Valmet: A New Validation Tool for Assessing and Improving 3D Object Segmentation. In In: MICCAI 2001: Fourth International Conference 
on Medical Image Computing and Computer-Assisted Intervention, 516-523. Springer.

Gu X, Wang Y, Chan T. F, Thompson PM, and Yau ST. 2004. Genus zero surface conformal mapping and its application to brain surface mapping. Medical Imaging, IEEE Transactions on 23, no. 8 (August): 949-958. doi:10.1109/TMI.2004.831226.

Han X, Xu C, Braga-Neto U, and Prince JL. 2002. Topology correction in brain cortex segmentation using a multiscale, graph-based algorithm. IEEE Transactions on Medical Imaging 21, no. 2: 109-121.

Hellier P, Barillot C, Corouge I, Gibaud B, Le Goualher G, Collins DL, Malandain G, Ayache N, Christensen GE, and Johnson HJ. 2003. Retrospective evaluation of intersubject brain registration. IEEE Transactions on Medical Imaging 22, no. 9: 1120-1130.

Hong W, Gu X, Qiu F, Jin M, and Kaufman AE. 2006. Conformal virtual colon flattening. In Symposium on Solid and Physical Modeling, 85-93.

Hua X, Leow AD, Lee S, Klunder AD, Toga AW, Lepore N, Chou YY, et al. 2008. 3D characterization of brain atrophy in Alzheimer's disease and mild cognitive impairment using tensor-based morphometry. Neurolmage 41, no. 1 (May): 19-34. doi:http://dx.doi.org/10.1016/j.neuroimage.2008.02.010.

Jaume S, Rondao P, and Macq B. 2005. Open Topology: A Toolkit for Brain Isosurface Correction. In MICCAI Open Source Workshop.

Kawachi T, Ishii K, Sakamoto S, Sasaki M, Mori T, Yamashita F, Matsuda H, and Mori E. 2006. Comparison of the diagnostic performance of FDG-PET and VBM-MRI in very mild Alzheimer's disease. European Journal of Nuclear Medicine and Molecular Imaging 33, no. 7 (July): 801-809.

Klauschen F, Goldman A, Barra V, Meyer-Lindenberg A, and Lundervold A. 2009. Evaluation of Automated Brain MR Image Segmentation and Volumetry Methods. Human Brain Mapping. No. 30: 1310-1327.

Kim JS, Singh V, Lee JK, Lerch J, Ad-Dab'bagh Y, MacDonald D, Lee JM, Kim SI, and Evans AC. 2005. Automated 3D extraction and evaluation of the inner and outer cortical surfaces using a Laplacian map and partial volume effect classification. Neurolmage 27, no. 1 (August): 210-221.

Kriegeskorte N, and Goebel R. 2001. An Efficient Algorithm for Topologically Correct Segmentation of the Cortical Sheet in Anatomical MR Volumes. Neuroimage 14: 329346.

Kuhn HW. 1955. The Hungarian method for the assignment problem. Naval Research Logistics Quarterly, no. 2: 83-87.

Lerch JP, and Evans AC. 2005. Cortical thickness analysis examined through power analysis and a population simulation. Neuroimage 24, no. 1 (January): 163-173.

Lerch JP, Pruessner JC, Zijdenbos A, Hampel H, Teipel SJ, and Evans AC. 2005. Focal decline of cortical thickness in Alzheimer's disease identified by computational neuroanatomy. Cerebral Cortex 15, no. 7 (July): 995-1001.

Lorensen WE, and Cline HE. 1987. Marching cubes: A high resolution 3D surface construction algorithm.

Mangin JF, Rivière D, Cachia A, Duchesnay E, Cointepas $Y$, Papadopoulos-Orfanos D, Scifo P, Ochiai T, Brunelle F, and Règis J. 2004. A framework to study the cortical folding patterns. Neurolmage 23, no. Supplement 1: S129 - S138. doi:DOI: 
10.1016/j.neuroimage.2004.07.019.

Mangin JF, Frouin V, Bloch I, Régis J, and López-Krahe J. 1995. From 3D magnetic resonance images to structural representations of the cortex topography using topology preserving deformations. Journal of Mathematical Imaging and Vision 5, no. 4 (December): 297318.

Marcus DS, Wang TH, Parker J, Csernansky JG, Morris JC, and Buckner RL. 2007. Open Access Series of Imaging Studies (OASIS): Cross-sectional MRI Data in Young, Middle Aged, Nondemented, and Demented Older Adults. Journal of Cognitive Neuroscience 19, no. 9: 1498-1507. doi:10.1162/jocn.2007.19.9.1498.

McKhann G, Drachman D, Folstein M, Katzman R, Price D and Stadlan E.M. 1984. Clinical Diagnosis of Alzheimer's Disease: report of the NINCDS-ADRDA Work Group under the auspices of Department of Health and Human Services Task Force on Alzheimer's Disease. Neurology, 34 , pp. 939-944

Mikhno A, Devanand D, Pelton G, Cuasay K, Gunn R, Upton N, Lai RY, Libri V, Mann JJ, and Parsey RV. 2008. Voxel-based analysis of 11C-PIB scans for diagnosing Alzheimer's disease. The Journal of Nuclear Medicine 49, no. 8 (August): 1262-1269.

Mori G, Belongie S, and Malik J. 2005. Efficient shape matching using shape contexts. Pattern Analysis and Machine Intelligence, IEEE Transactions on 27, no. 11 (November): 18321837. doi:10.1109/TPAMI.2005.220.

Petersen RC, Smith GE, Waring SC, Ivnik RJ, Tangalos EG and Kokmen E 1999. Mild cognitive impairment: clinical characterization and outcome. Arch. Neurol, 56 , pp. 303-308.

Pons JP, Keriven R, and Faugeras O. 2004. Area Preserving Cortex Unfolding. In Medical Image Computing and Computer-Assisted Intervention - MICCAI 2004, ed. David R. Haynor Christian Barillot and Pierre Hellier (Eds.), 3216:376-383. LNCS. Springer.

Querbes, O, Aubry F, Pariente J, Lotterie JA, Demonet JF, Duret V, Puel M, et al. 2009. Early diagnosis of Alzheimer's disease using cortical thickness: impact of cognitive reserve. Brain 132, no. 8: 2036-2047. doi:10.1093/brain/awp105.

Rueckert D, Sonoda LI, Hayes C, Hill DLG, Leach MO, and Hawkes DJ. 1999. Nonrigid Registration Using Free-Form Deformations: Application to Breast MR Images. IEEE Transactions on Medical Imaging 18 (August): 712-721.

Rueda A, Acosta O, Bourgeat P, Fripp J, Dowson N, Couprie M, Romero E, and Salvado O. 2009. Partial Volume Estimation of Brain Cortex from MRI Using Topology-Corrected Segmentation. In 2009 IEEE International Symposium on Biomedical Imaging: From Nano to Macro, 133-136. Boston, Massachusetts, USA: IEEE, June.

Rueda, A.; Acosta, O.; Couprie, M.; Bourgeat, P.; Fripp, J.; Dowson, N.; Romero, E. and Salvado, O. Topology-corrected segmentation and local intensity estimates for improved partial volume classification of brain cortex in MRI. Journal of Neuroscience Methods, 2010, 188, 305-315.

Ségonne F. 2008. Active Contours Under Topology Control - Genus Preserving Level Sets. International Journal of Computer Vision 79, no. 2: 107 - 117.

Shi Y, Thompson PM, Dinov I, Osher S, and Toga AW. 2007. Direct cortical mapping via solving partial differential equations on implicit surfaces. Medical Image Analysis 11, no. 3: 207 - 223. doi:DOI: 10.1016/j.media.2007.02.001.

Tosun D, Rettman ME, and Prince JL. 2004. Mapping techniques for aligning sulci across 
multiple brains. Medical Image Analysis (5): 295-309.

Tosun D, Rettmann ME, Han X, Tao X, Xu C, Resnick SM, Pham DL, and Prince JL. 2004. Cortical surface segmentation and mapping. Neurolmage 23, no. Supplement 1: S108 - S118. doi:DOI: 10.1016/j.neuroimage.2004.07.042.

Tzourio-Mazoyer N, Landeau B, Papathanassiou D, Crivello F, Etard O, Delcroix N, Mazoyer B, and Joliot M. 2002. Automated anatomical labeling of activations in SPM using a macroscopic anatomical parcellation of the MNI MRI single-subject brain. Neuroimage 15, no. 1 (January): 273-289.

Urschler M, and Bischof H. 2004. Registering 3D Lung Surfaces Using the Shape Context Approach. Proc. Medical Image Understanding and Analysis: 212-215.

Van Leemput K, Maes F, Vandermeulan D, and Suetens P. 1999. Automated model-based bias field correction of MR images of the brain. IEEE Transactions on Medical Imaging 18, no. 10 (October): 885-896.

Vercauteren T, Pennec X, Perchant A, and Ayache N. 2007. Non-parametric Diffeomorphic image Resgistration with the Demons Algorithm. In Medical Image Computing and Computer-Assisted Intervention, 4792:319-326. LNCS. Brisbane, Australia: Springer Berlin / Heidelberg, October.

Villain N, Desgranges B, Viader F, de la Sayette V, Mezenge F, Landeau B, Baron JC, Eustache F, and Chetelat G. 2008. Relationships between Hippocampal Atrophy, White Matter Disruption, and Gray Matter Hypometabolism in Alzheimer's Disease. The Journal of Neuroscience 28, no. 24 (June): 6174-6181.

Yeo B, Thomas T, Sabuncu M, Vercauteren T, Ayache N, Fischl B, and Golland P. 2008. Spherical Demons: Fast Surface Registration. In Medical Image Computing and Computer-Assisted Intervention - MICCAI 2008, ed. Dimitris Metaxas, Leon Axel, Gabor Fichtinger, and Gábor Székely, 5241:745-753. LNCS. Springer.

Yeo B., Sabuncu M, Vercauteren T, Ayache,N, Fischl B., and Golland P. Spherical Demons: Fast Diffeomorphic Landmark-Free Surface Registration Medical Imaging, IEEE Transactions on, 2010, 29, 650 -668

Zhan J, Brys M, Glodzik L, Tsui W, Javier E, Wegiel J, Kuchna I, et al. 2009. An entorhinal cortex sulcal pattern is associated with Alzheimer's disease. Human Brain Mapping 30, no. 7: 874-882.

Zhou QY, Ju T, and Hu SM. 2007. Topology Repair of Solid Models Using Skeletons. IEEE Transactions on Visualization and Computer Graphics 13, no. 4: 675-685. doi:http://dx.doi.org/10.1109/TVCG.2007.1015.

Ziolko SK, Weissfeld LA et al. 2006. Evaluation of voxel-based methods for the statistical analysis of PIB PET amyloid imaging studies in Alzheimer's disease. Neuroimage 33, no. 1 (October): 94-102. 


\section{Algorithms}

Data: a mesh M

Result: M homeomorphic to a sphere

Foreach $b \in$ boundaries (M) do

$b \in$ boundaries (M) Close the surface on $b$ by adding a disc;

While genus $(M) \neq 0$ do

$I=$ an approximation of the shortest non-separating loop;

Cut $\mathrm{M}$ according to $\mathrm{l}$;

Close the surface on each side of the created boundaries by adding a disc;

Algorithm 1: Topological correction

Data: a mesh M non homeomorphic to a sphere

Result: a non-separating loop I

$B$ reduced cut locus;

Foreach: path $p \subset B$ do

$I p=$ shortest no-separating loop containing $p ;$

if $I$ not defined or length $(I p)<$ length $(I)$ then

I=Ip;

Algorithm 2 : Non-separating loop detection 


\section{TABLES}

Table 1: Average percentage of vertices with $\hat{M}_{A}$ in range $[0.8,1.2]$

\begin{tabular}{|l|l|l|l|}
\hline \multicolumn{2}{|c|}{ Hemisphere } & CARET & FreeSurfer \\
\hline Left & Frontal & 49.31 & 38.67 \\
\hline & Temporal & 45.77 & 37.69 \\
\hline & Occipital & 39.94 & 31.92 \\
\hline & Parietal & 46.67 & 32.86 \\
\hline Right & Total & 42.41 & 32.94 \\
\hline & Frontal & 49.59 & 39.00 \\
\hline & Temporal & 45.13 & 37.49 \\
\hline & Occipital & 39.50 & 32.91 \\
\hline & Parietal & 46.76 & 32.44 \\
\hline & Total & 42.47 & 32.91 \\
\hline
\end{tabular}

Table 2: Averaged Hausdorff Distance (HD), Mean Absolute Distance (MAD) and area relation (\% A $M-F$ ) betweeen moving $M$ and fixed $F$ surfaces after shape context registration. Mean (standard deviation), as the number of sampling control points varies. 30 left and 30 right hemispheres.

\begin{tabular}{|c|c|c|c|c|c|c|}
\hline & & left $\mathbf{h}$ & & & right $\mathbf{h}$ & \\
\hline NPts & 500 & 1000 & 2000 & 500 & 1000 & 2000 \\
\hline HD [mm] & $8.45(1.9)$ & $7.25(1.97)$ & $6.29(1.71)$ & 14.97 & 13.24 & 13.11 \\
& & & & $(7.13)$ & $(7.83)$ & $(8.25)$ \\
\hline MAD [mm] & $0.74(0.05)$ & $0.49(0.04)$ & $0.35(0.03)$ & $1.17(0.58)$ & $0.86(0.61)$ & $0.74(0.83)$ \\
\hline$\% A$ & $98.08 \%$ & $98.45 \%$ & $98.72 \%$ & $97.02 \%$ & $97.72 \%$ & $98.8 \%$ \\
\hline
\end{tabular}


Table 3: Averaged (standard deviation) jaccard coefficient on some anatomical labels computed after registration with Shape Context (SC) and with Freesurfer (FS).

\begin{tabular}{|l|l|c|c|c|c|}
\hline \multicolumn{2}{|c|}{} & \multicolumn{2}{c|}{ SC } & \multicolumn{2}{c|}{ FS } \\
\hline Region & Hemisphere & Average & St Dev & Average & St Dev \\
\hline 5_cuneus & Left & 0,54 & 0,08 & 0,66 & 0,06 \\
\hline 5_cuneus & Right & 0,62 & 0,08 & 0,55 & 0,18 \\
\hline 7_fusiform & Left & 0,55 & 0,10 & 0,58 & 0,13 \\
\hline 7_fusiform & Right & 0,60 & 0,08 & 0,58 & 0,10 \\
\hline 10_isthmuscingulate & Left & 0,36 & 0,10 & 0,46 & 0,09 \\
\hline 10_isthmuscingulate & Right & 0,54 & 0,11 & 0,51 & 0,08 \\
\hline 11_lateraloccipital & Left & 0,66 & 0,04 & 0,63 & 0,06 \\
\hline 11_lateraloccipital & Right & 0,62 & 0,06 & 0,53 & 0,08 \\
\hline 12_lateralorbitofrontal & Left & 0,77 & 0,03 & 0,68 & 0,06 \\
\hline 12_lateralorbitofrontal & Right & 0,76 & 0,05 & 0,71 & 0,04 \\
\hline 17_paracentral & Left & 0,69 & 0,06 & 0,71 & 0,07 \\
\hline 17_paracentral & Right & 0,65 & 0,08 & 0,68 & 0,12 \\
\hline 20_parstriangularis & Left & 0,42 & 0,09 & 0,60 & 0,09 \\
\hline 20_parstriangularis & Right & 0,46 & 0,12 & 0,59 & 0,09 \\
\hline 24_precentral & Left & 0,71 & 0,06 & 0,82 & 0,03 \\
\hline 24_precentral & Right & 0,73 & 0,05 & 0,68 & 0,14 \\
\hline 25_precuneus & Left & 0,69 & 0,05 & 0,78 & 0,04 \\
\hline 25_precuneus & Right & 0,71 & 0,05 & 0,70 & 0,08 \\
\hline 30_superiortemporal & Left & 0,69 & 0,05 & 0,77 & 0,03 \\
\hline 30_superiortemporal & Right & 0,67 & 0,06 & 0,72 & 0,05 \\
\hline 34_transversetemporal & Left & 0,11 & 0,76 & 0,07 \\
\hline 34_transversetemporal & Right & 0,11 & 0,70 & 0,11 \\
\hline
\end{tabular}


Table 4: Average (standard deviation) cortical thickness, computed with milxCTE and Freesurfer and correlation between both methods in the AAL Regions.

\begin{tabular}{|c|c|c|c|c|c|c|}
\hline \multirow[b]{2}{*}{ Structure } & \multicolumn{2}{|c|}{ milxCTE } & \multicolumn{2}{|c|}{ Freesurfer } & \multicolumn{2}{|c|}{ Comparison } \\
\hline & Average(mm) & St Dev & Average $(\mathrm{mm})$ & St Dev & $\begin{array}{c}\text { Difference } \\
(\mathrm{mm})\end{array}$ & $r$ \\
\hline Supramarginal L & 2.38 & 0.20 & 2.45 & 0.13 & 0.07 & 0.69 \\
\hline Supramarginal $\mathrm{R}$ & 2.38 & 0.17 & 2.45 & 0.14 & 0.07 & 0.65 \\
\hline Superiorfrontal L & 2.49 & 0.17 & 2.59 & 0.12 & 0.01 & 0.64 \\
\hline Superiorfrontal R & 2.54 & 0.20 & 2.54 & 0.11 & 0 & 0.73 \\
\hline Rostralmiddlefrontal L & 2.32 & 0.18 & 2.28 & 0.11 & -0.04 & 0.72 \\
\hline Rostralmiddlefrontal $\mathrm{R}$ & 2.36 & 0.18 & 2.25 & 0.10 & -0.11 & 0.67 \\
\hline $\begin{array}{l}\text { Rostralanteriorcingulate } \\
\text { L }\end{array}$ & 2.62 & 0.32 & 2.64 & 0.21 & 0.02 & 0.68 \\
\hline $\begin{array}{l}\text { Rostralanteriorcingulate } \\
\text { R }\end{array}$ & 2.67 & 0.29 & 2.52 & 0.18 & -0.15 & 0.78 \\
\hline Precuneus L & 2.53 & 0.27 & 2.28 & 0.15 & -0.25 & 0.79 \\
\hline Precuneus $\mathrm{R}$ & 2.45 & 0.26 & 2.28 & 0.14 & -0.17 & 0.8 \\
\hline Precentral L & 2.21 & 0.28 & 2.43 & 0.13 & 0.22 & 0.72 \\
\hline Precentral R & 2.26 & 0.27 & 2.38 & 0.15 & 0.12 & 0.83 \\
\hline Posteriorcingulate $\mathrm{L}$ & 2.57 & 0.24 & 2.38 & 0.14 & -0.19 & 0.57 \\
\hline Posteriorcingulate $\mathrm{R}$ & 2.49 & 0.21 & 2.33 & 0.12 & -0.16 & 0.72 \\
\hline Pericalcarine $\mathrm{L}$ & 2.29 & 0.29 & 1.74 & 0.12 & -0.55 & 0.65 \\
\hline Pericalcarine $\mathrm{R}$ & 2.2 & 0.25 & 1.76 & 0.12 & -0.44 & 0.72 \\
\hline Pars triangularis L & 2.41 & 0.15 & 2.32 & 0.11 & -0.09 & 0.65 \\
\hline Pars triangularis $\mathrm{R}$ & 2.3 & 0.16 & 2.34 & 0.12 & -0.04 & 0.62 \\
\hline Inferior temporal L & 2.87 & 0.36 & 2.67 & 0.15 & -0.2 & 0.6 \\
\hline
\end{tabular}




\begin{tabular}{|l|c|c|c|c|c|l|}
\hline Inferior temporal R & 2.76 & 0.29 & 2.77 & 0.18 & -0.01 & 0.55 \\
\hline Inferior parietal L & 2.48 & 0.24 & 2.38 & 0.14 & -0.10 & 0.86 \\
\hline Inferior parietal R & 2.35 & 0.22 & 2.39 & 0.15 & 0.04 & 0.86 \\
\hline Superiorfrontal L & 2.45 & 0.17 & 2.59 & 0.12 & 0.14 & 0.80 \\
\hline Superiorfrontal R & 2.50 & 0.20 & 2.54 & 0.11 & 0.04 & 0.75 \\
\hline
\end{tabular}




\section{Figure Legends}

Figure 1: Overall pipeline for cortical mapping with milxCTE. After WM/GM segmentation (1-4), topology is corrected on each separated hemisphere (5), partial inflation is performed (6) and eventually surfaces are registered towards a common coordinate system (7).

Figure 2: Cutting and patching of non-separating loops. a) Computation of the cut locus B associated to a point $p$ on a 1-genus surface M. b) Cut locus B associated to a point $p$ on a 2genus surface M. c) Computation of the shortest non-separating loop associated to a point on the path $\mathrm{S}_{\mathrm{B}}$, subpart of the cut locus $\mathrm{B}$ associated to the point $p$.

Figure 3: Example of topological correction of $a, b)$ a tunnel, c,d) two handles.

Figure 4: Top: Original cortical mesh is flattened to a PFS and color coded sulcal depth. (Eq. 2) is mapped over each point in the surface as inflation progresses (20,50 and 100 iterations). a) Original Mesh b) 20 iterations c) 50 iterations d) 100 iterations. Bottom : Local Area distortion map after inflation using CARET and Freesurfer.

Figure 5: Finding correspondences between two individuals. After PFS are obtained, the surface $M$ is registered towards the fixed $F$ target. $M S$ and $F S$ are the control points to match.

Figure 6: Propagated labels from the template d) to the patient c) after the registration of both PFS surfaces a), b).

Figure 7: Regional mapping of the correlation $(r)$ between cortical thickness computed with both methods: milxCTE and Freesurfer, left and right hemispheres.

Figure 8: Example of correlations in some regions left/right hemispheres. a,b) SFG: superior frontal gyrus and c,d) IPG: Inferior parietal gyrus [SFG (left H), $r=0.80$ ] [SFG (right H), $r$ $=0.75$ ] [IPG (left H), $r=0.86]$ [IPG (right H), $r=0.86$ ] (corrected values) 
Figure 9: Top: Resulting AAL labelling after voting using a population labels mapped to a common template. Right and left hemispheres, lateral and medial views. Middle: Cortical thickness mapped onto a common template: a HC individual, bottom: an AD patient. The pronounced generalized atrophy in $A D$ compared to a $\mathrm{HC}$ is visible, mainly in temporal areas.

Figure 10: Top: Vortex-wise differences in averaged cortical thickness between the two groups AD and HC. Bottom: Statistical p-values Map after two sampled t-test. 


\section{T1W-MRI}

1. Pure tissue Segmentation (WM,GM,CSF)

2. Partial volume classification (WM/GM,GM/CSF)

3. Binary topology correction

- Homotopic dilation of WM over GM

4. Hemispheres separation

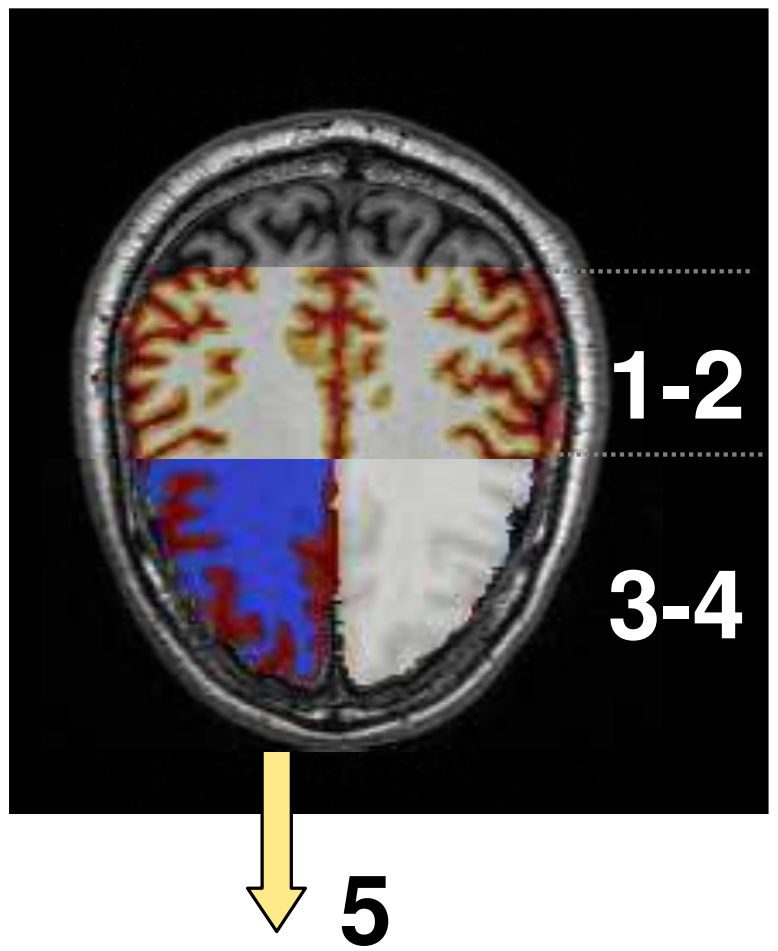

5. Genus zero surface generation
6. Surface inflation

7. Non rigid registration

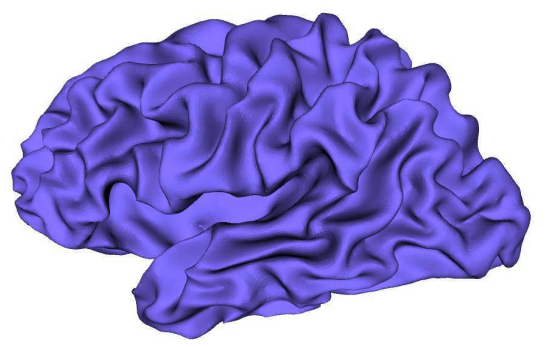

\ 6

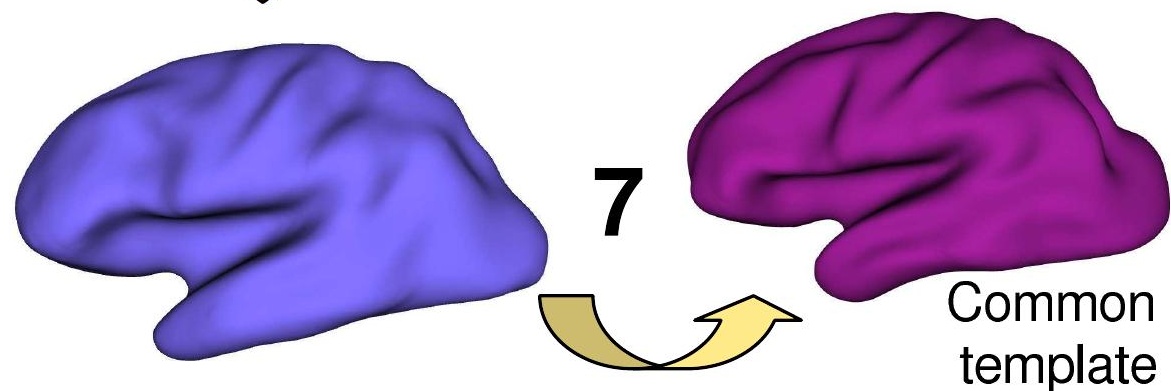



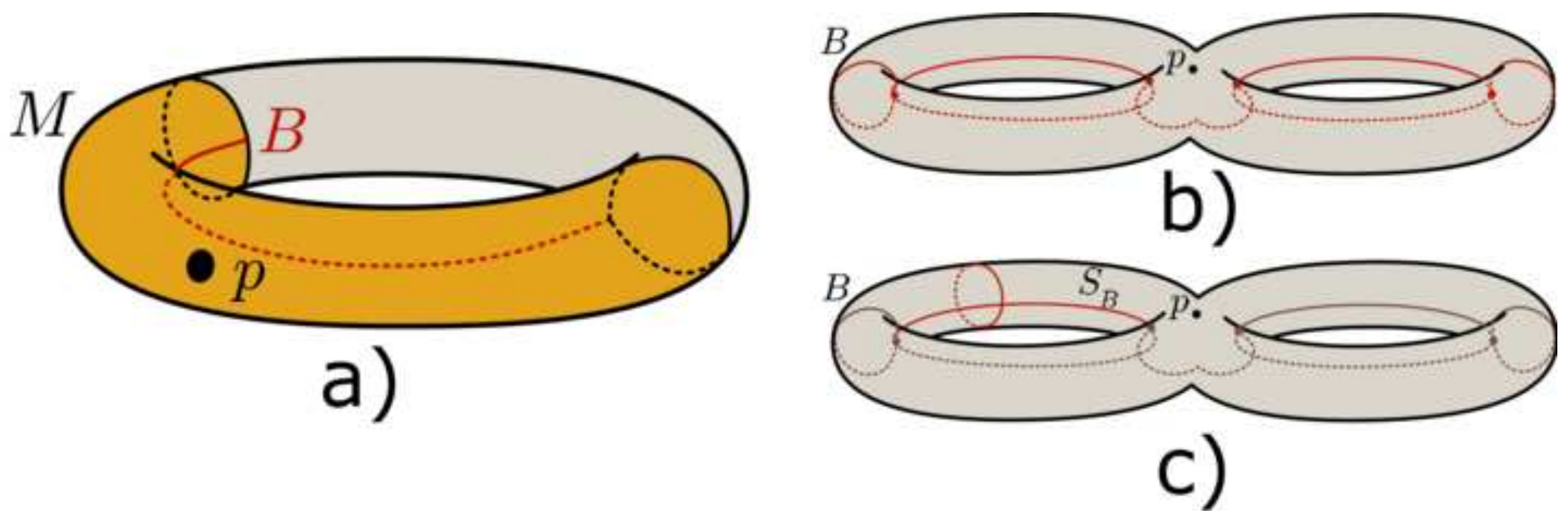
Click here to download high resolution image
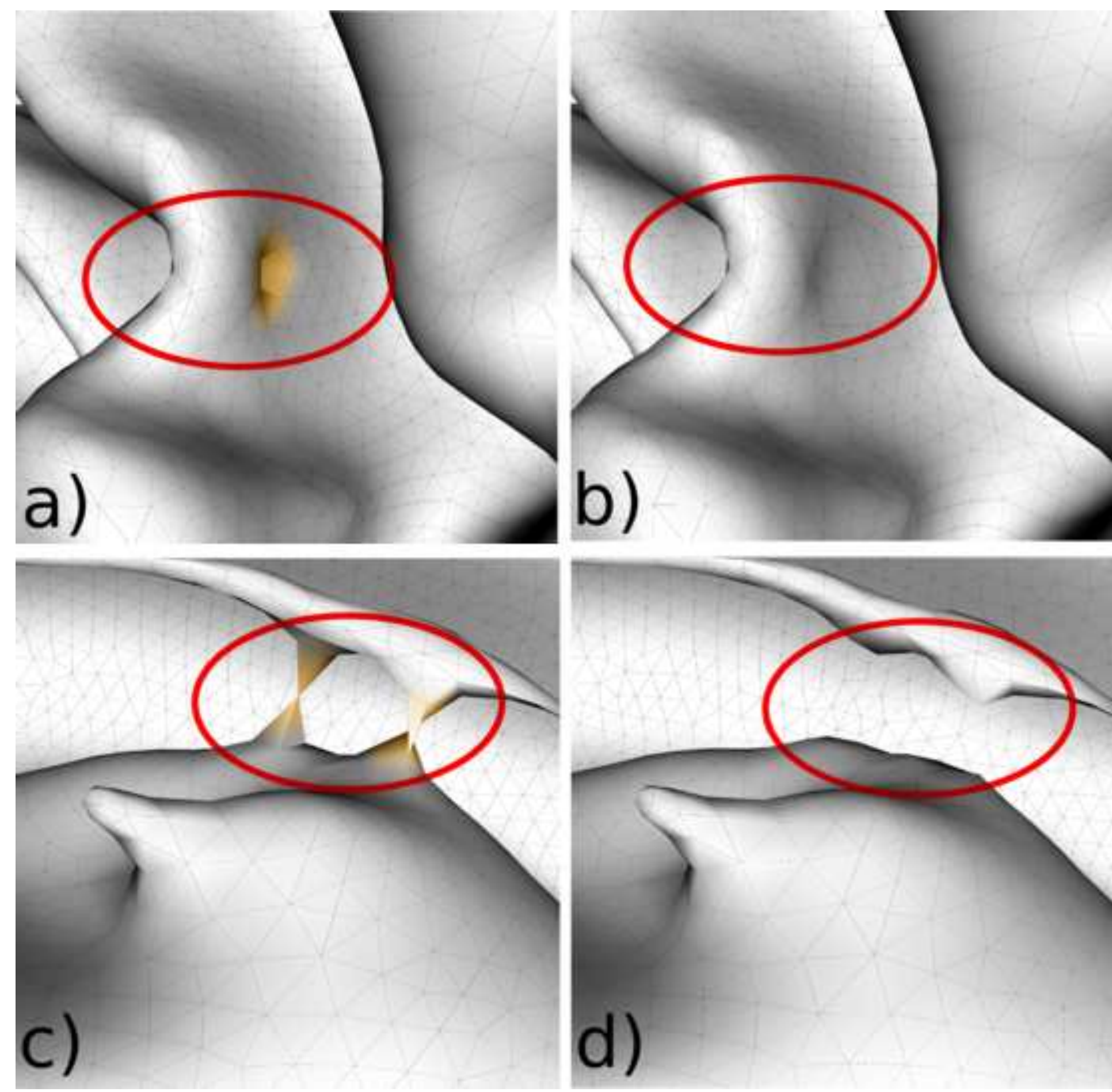

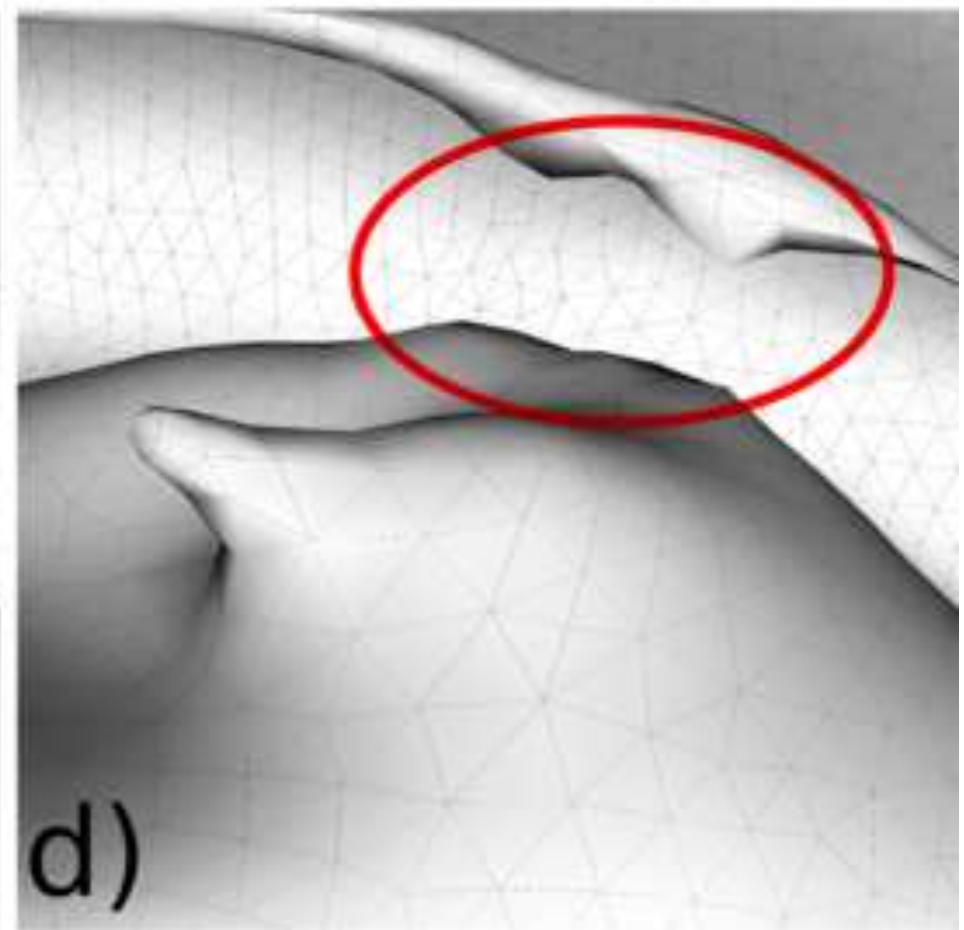

b)

a) 


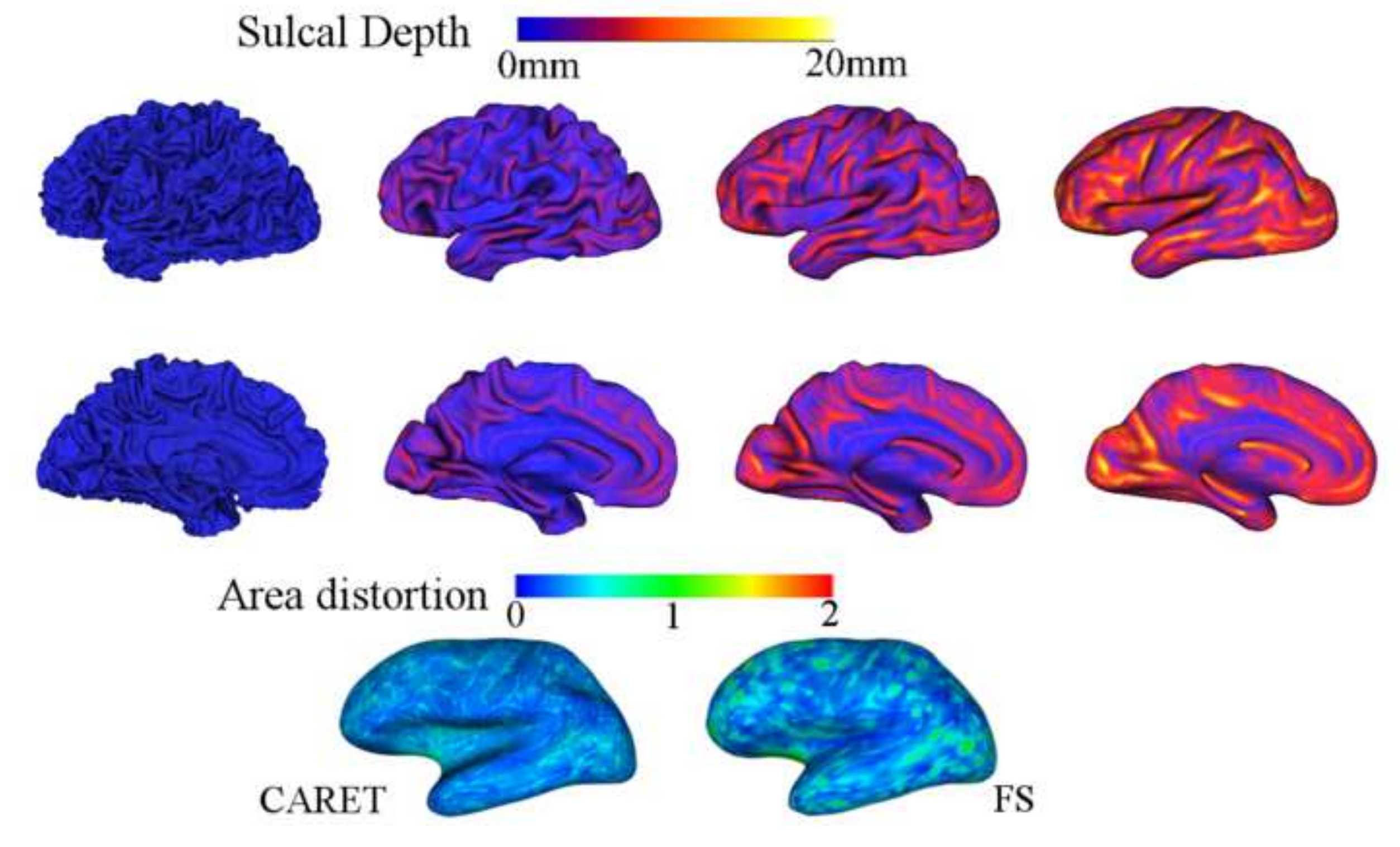

Area distortion $\begin{array}{lll} & & \\ 0 & & 1\end{array}$

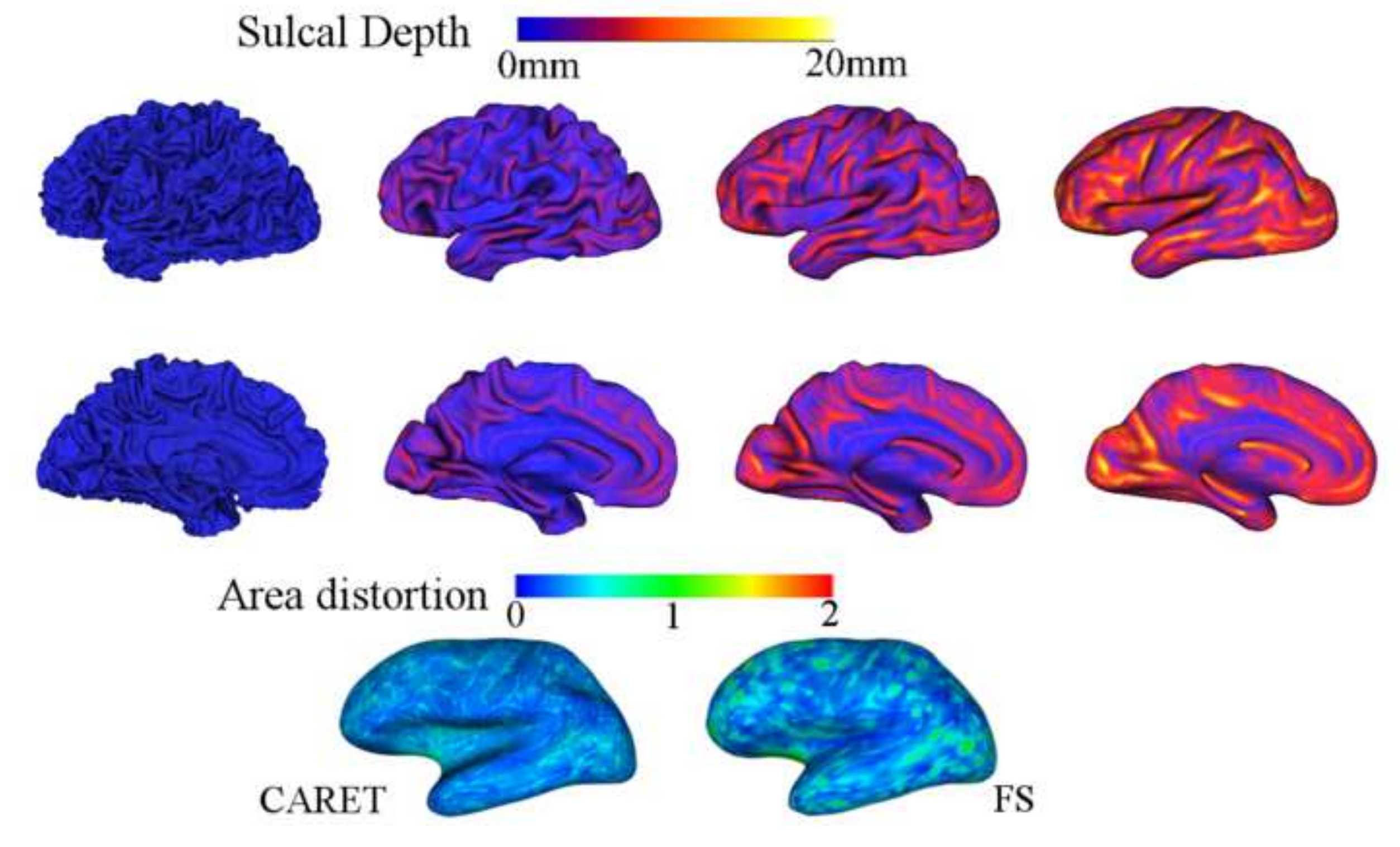

Click here to download high resolution image 

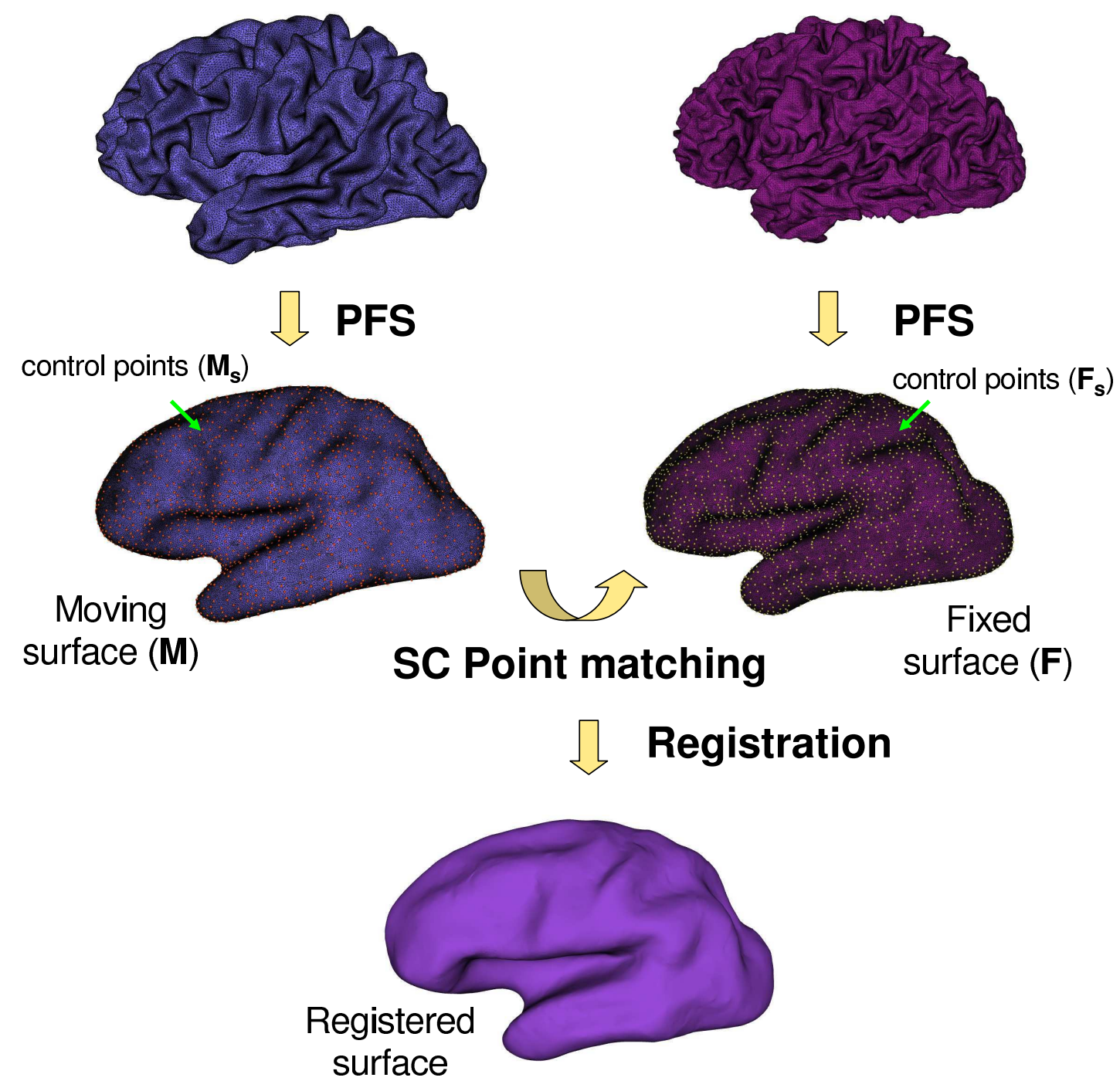

surface

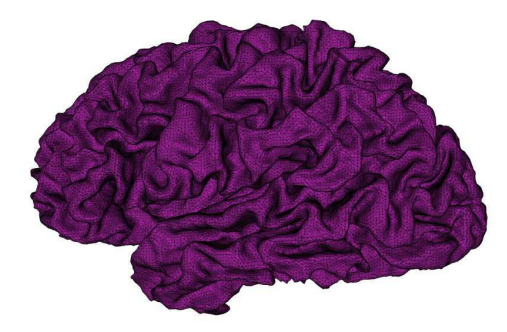

surface (F) 
Figure 6

(a)

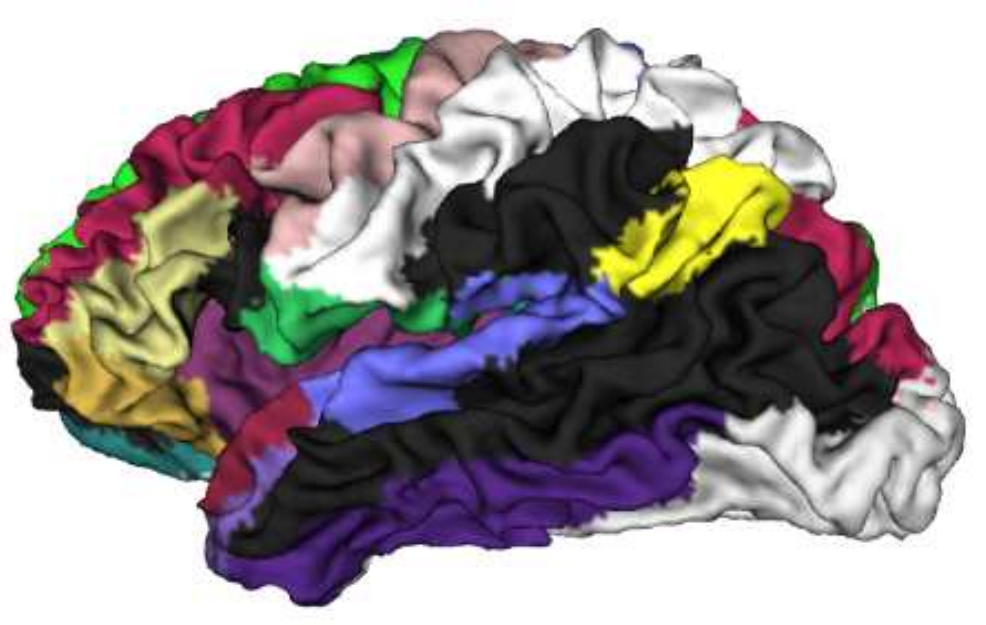

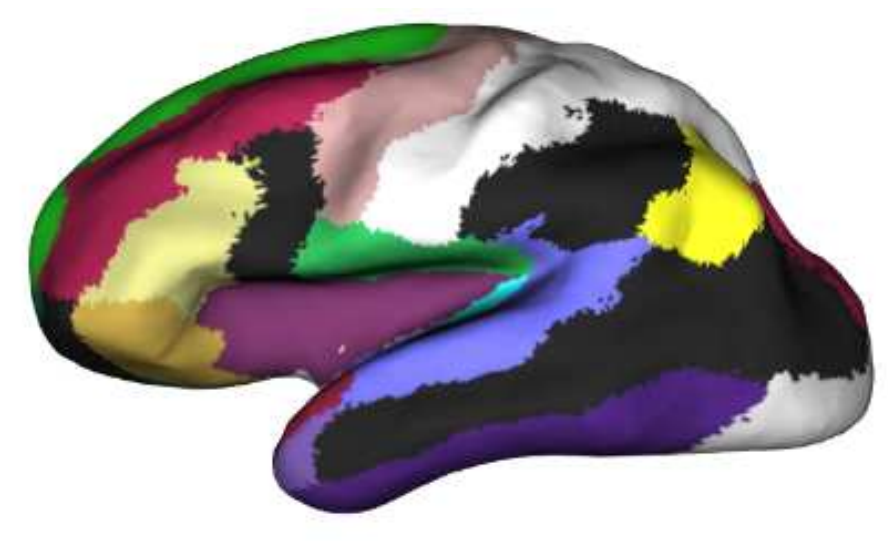

(b)

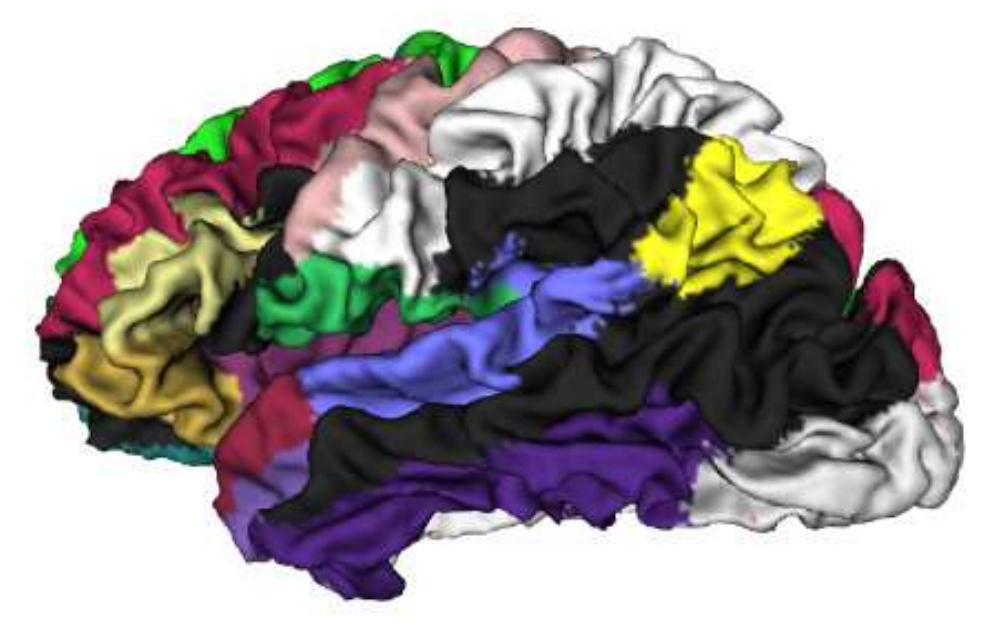

(c)

(d) 


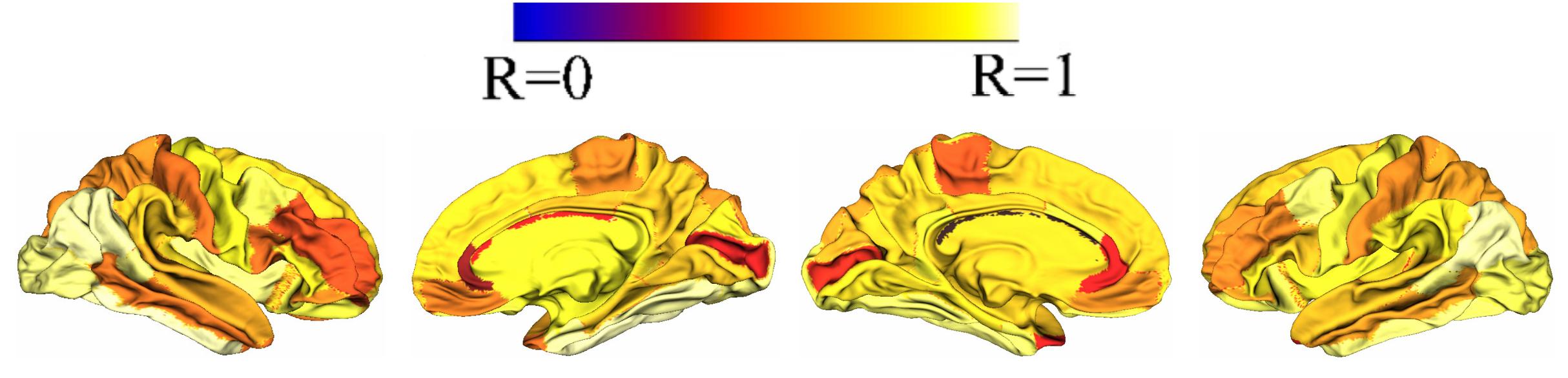



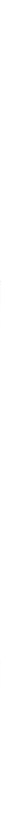

\section{FS}
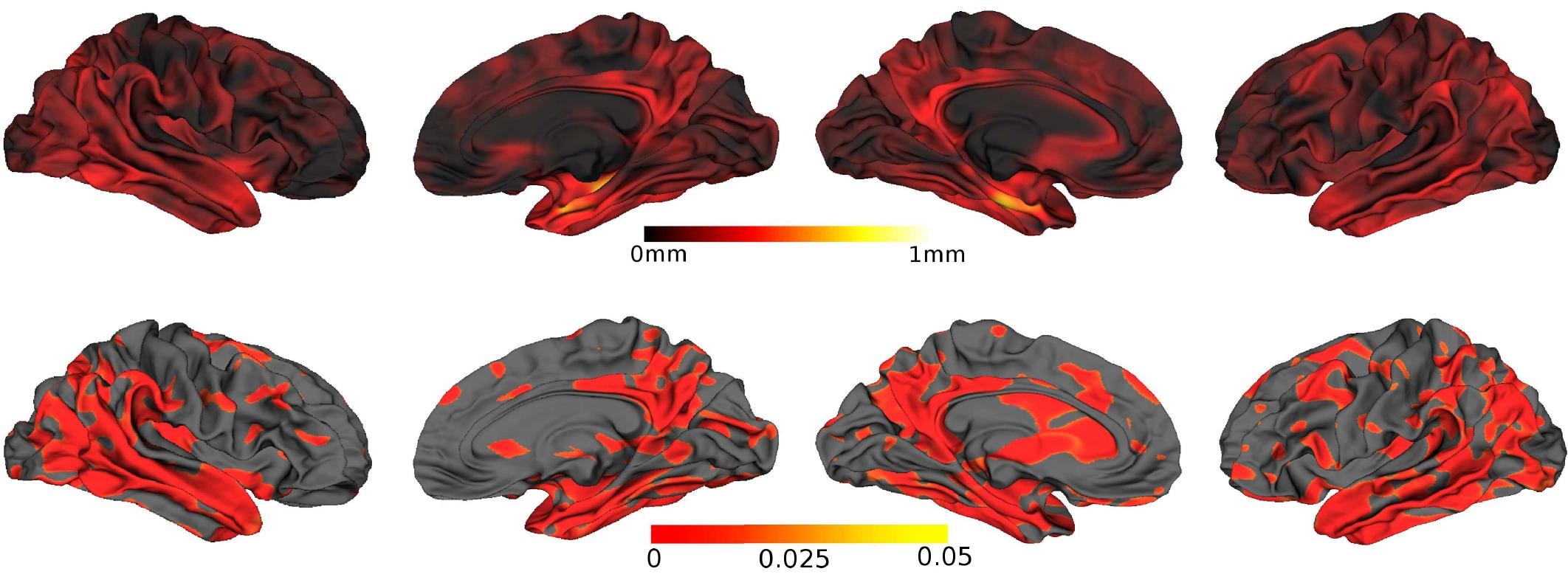

Supplementary file for online publication only
Click here to download Supplementary file for online publication only: BJPFSMedial.avi . (1) (1)

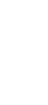
(1) (1) . . . . . . . . . . . . . 